\title{
High-Resolution Mapping of Flows in the Solar Interior: Fully Consistent OLA Inversion of Helioseismic Travel Times
}

\author{
J. Jackiewicz • L. Gizon • A.C. Birch
}

Received: 17 October 2007 / Accepted: 29 February 2008 / Published online: 9 April 2008

(C) The Author(s) 2008. This article is published with open access at Springerlink.com

\begin{abstract}
To recover the flow information encoded in travel-time data of time-distance helioseismology, accurate forward modeling and a robust inversion of the travel times are required. We accomplish this using three-dimensional finite-frequency travel-time sensitivity kernels for flows along with a $(2+1)$-dimensional $(2+1 \mathrm{D})$ optimally localized averaging (OLA) inversion scheme. Travel times are measured by ridge filtering MDI full-disk Doppler data and the corresponding Born sensitivity kernels are computed for these particular travel times. We also utilize the full noise-covariance properties of the travel times, which allow us to accurately estimate the errors for all inversions. The whole procedure is thus fully consistent. Because of ridge filtering, the kernel functions separate in the horizontal and vertical directions, motivating our choice of a $2+1 \mathrm{D}$ inversion implementation. The inversion procedure also minimizes cross-talk effects among the three flow components, and the averaging kernels resulting from the inversion show very small amounts of cross-talk. We obtain three-dimensional maps of vector solar flows in the quiet Sun at horizontal spatial resolutions of $7-10 \mathrm{Mm}$ using generally 24 hours of data. For all of the flow maps we provide averaging kernels and the noise estimates. We present examples to test the inferred flows, such as a comparison with Doppler data, in which we find a correlation of 0.9. We also present results for quiet-Sun supergranular flows at different depths in the upper convection zone. Our estimation of the vertical velocity shows good qualitative agreement with the horizontal vector flows. We also show vertical flows measured solely from $f$-mode travel times. In addition, we demonstrate how to directly invert for the horizontal divergence and
\end{abstract}

Helioseismology, Asteroseismology, and MHD Connections

Guest Editors: Laurent Gizon and Paul Cally.

J. Jackiewicz $(\bowtie) \cdot$ L. Gizon

Max-Planck-Institut für Sonnensystemforschung, 37191 Katlenburg-Lindau, Germany

e-mail: jackiewicz@mps.mpg.de

L. Gizon

e-mail: gizon@mps.mpg.de

A.C. Birch

NWRA CoRA Division, Boulder, CO 80301, USA

e-mail: aaronb @cora.nwra.com 
flow vorticity. Finally we study inferred flow-map correlations at different depths and find a rapid decrease in this correlation with depth, consistent with other recent local helioseismic analyses.

Keywords Helioseismology · Inverse modeling · Velocity fields · Photosphere · Supergranulation

\section{Introduction}

Time-distance helioseismology (Duvall et al., 1993) comprises a set of tools that measures and interprets the travel times of seismic waves propagating from one point on the solar surface to any other point. It has been shown that these travel times contain information about solar flows (Kosovichev, 1996; Duvall et al., 1997; Duvall and Gizon, 2000; Gizon, Duvall, and Larsen, 2000; Zhao, Kosovichev, and Duvall, 2001, among others). This paper focuses on the inversion of travel times to obtain high-spatial-resolution maps of near-surface vector flows in quiet-Sun regions. What is unique to this study is that it is the first fully consistent inversion in time-distance helioseismology. The consistency is described by several factors: $i$ ) We measure travel times with the same definition with which the travel-time sensitivity kernels are computed; $i$ ) we use three-dimensional (3D) finitefrequency Born sensitivity kernels, which are necessary to detect flow structures that have spatial scales on the order of the mode wavelength - the regime where the commonly used ray approximation fails (Birch and Felder, 2004, for example); iii) we use the full noisecovariance properties of the travel times as an ingredient in the inversion; $i v$ ) the inversion procedure we choose to implement is "optimal," in that it simultaneously achieves the best possible spatial resolution while minimizing the magnification of the errors. Furthermore, the regularization is carried out in both the horizontal and vertical directions.

We have developed a novel two-plus-one-dimensional $(2+1 \mathrm{D})$ inversion scheme based on the well-known subtractive optimally localized averages (SOLA) technique (Pijpers and Thompson, 1992; Jackiewicz et al., 2007b). For our purposes, $2+1 \mathrm{D}$ refers to first solving a 2D horizontal inverse problem followed by a $1 \mathrm{D}$ inversion in depth. The inversion procedure explicitly minimizes the cross-talk effects among the three flow components by imposing constraints on the averaging kernels. An important aspect that we introduce to this procedure is that of ridge-filtered travel-time measurements, whereby only wave packets of a particular radial order are used. Sets of point-to-annulus travel times are then computed for the $f, p_{1}, p_{2}, p_{3}$, and $p_{4}$ ridges. This is quite different than the usual phase-speed filtering implemented for travel-time measurements. Subsequently, the sensitivity kernels are also computed as corresponding ridge-filtered quantities to match the travel times. The $2+1 \mathrm{D}$ inversion is motivated by the observation that to a very good approximation the $3 \mathrm{D}$ Born ridge-filtered sensitivity kernels separate into the product of a $2 \mathrm{D}$ horizontal function and a $1 \mathrm{D}$ function in depth.

Together, these ingredients allow us to infer all three components of the vector flow in the near-surface layers of the quiet-Sun convection zone. In previous work, we determined for the first time the maximum amplitude of flows that can be reliably recovered from a linear model of the travel-time perturbations (Jackiewicz et al., 2007a). The supergranular and other quiet-Sun flows (with velocities $\leq 400 \mathrm{~m} \mathrm{~s}^{-1}$ ) that we detect in this study fall within this range, giving us confidence in the reliability of the method. The horizontal spatial resolution of the inversion presented here $(\approx 7-10 \mathrm{Mm}$, depending on the observation time, depth, etc.) is on the order of, or even in some cases below, the wavelength of the waves used in the analysis (typically 5-20 Mm, depending on the dominant modes). In addition, 
we perform a direct measurement of the vertical component of the flow (without simply invoking mass conservation) and with confidence that the cross-talk between the horizontal and vertical components has been minimized. Interestingly, we find that we can determine the vertical velocity even from $f$-mode travel times. We also directly invert for the horizontal divergence of the flow as well as the vertical component of the flow vorticity.

As the main aim of this paper is to develop the inversion procedure and to perform tests of it on real solar data; we postpone the main interpretation of the results, as well as inversions carried out on artificial data, to a future publication. We typically show quiet-Sun flows that have been obtained from 24 hours of data, to maximize the signal-to-noise ratio from the supergranulation signal (Gizon and Birch, 2004). In all of the inferred flow maps we provide estimates of the noise and the spatial resolution.

The paper is organized as follows: In Section 2 we describe the data and the ridge-filtered travel-time measurements. This is followed in Section 3 by a brief discussion of the forward modeling, in other words, the computation of sensitivity kernels and the noise covariances that are consistent with the travel times. Since the multistep inversion procedure is somewhat complicated, we provide an overview in Section 4, followed by two sections that discuss in detail the 2D and 1D parts with several example calculations. Three-dimensional averaging kernels at different depths are presented in Section 7, and finally the various flow maps are presented, tested, and discussed in Section 8. We end with a summary of the results and a discussion of current and future work.

\section{Data and Ridge-Filtered Travel-Time Measurements}

For this study we use Dopplergram data from the Michelson Doppler Imager (MDI) (Scherrer et al., 1995) onboard SOHO, which are full-disk images of $0.12^{\circ}$ spatial sampling $(\approx 1.46 \mathrm{Mm})$ and one-minute cadence. The region of interest is an area centered on NOAA AR 9787 observed between 20 and 28 January 2002 and tracked and remapped courtesy of T.L. Duvall Jr. (To download these data as well as the corresponding magnetograms and intensity images for analysis, visit http://www.mps.mpg.de/projects/seismo/NA4/DATA/data access.html). The size of the full set of velocity data cubes is $512 \times 512 \times 1440 \times 9$ (two spatial dimensions, 1440 minutes, and nine days). We only use the middle seven days for the results shown here. These data are ideal for helioseismic analysis as there is a sunspot that is large, isolated, and quite stable as it traverses the disk. For our purposes, there are also large regions of relatively quiet Sun in these maps where we will focus our analysis.

We denote a Doppler velocity cube as $\phi(\mathbf{r}, t)$, where

$$
\mathbf{r}=(x, y)
$$

is the horizontal coordinate, $x$ and $y$ are the East-West and North-South directions, respectively, $t$ is time, and we work in a Cartesian geometry. We filter the data by multiplying the Fourier transform of the data cube by a filter $\left[F_{n}(\mathbf{k}, \omega)\right]$ that selects all modes with the same radial order $[n]$ and removes all others. We call this ridge filtering. The ridge-filtered data $\left[\Phi_{n}\right]$ are then given by

$$
\Phi_{n}(\mathbf{k}, \omega)=F_{n}(\mathbf{k}, \omega) \phi(\mathbf{k}, \omega)
$$

where $\mathbf{k}$ is the horizontal wavevector and $\omega$ is the angular frequency. The ridges we filter and retain for this study are the surface-gravity wave ( $f$-mode) ridge and the first four acoustic ( $p$-mode) ridges. Throughout the text we carry the index $n$, which takes the possible values 
$n=\left\{f, p_{1}, p_{2}, p_{3}, p_{4}\right\}$. We refer to these as mode ridges. It is important to note that this type of filtering is different from the phase-speed filtering that is typically done prior to any time-distance analysis.

The cross-covariance functions are computed from $\Phi_{n}(\mathbf{k}, \omega)$ for three different pointto-annulus geometries, denoted by "oi" (out minus in), "we" (West minus East), and "ns" (North minus South). The "oi" covariances are measured by using the wave signal at a given point and the wave signal averaged over a concentric annulus of radius $\Delta$. The "we" ("ns") quantities use the central wave signal along with the wave signal averaged over the annulus but weighted by $\cos \theta(\sin \theta)$, where $\theta$ is the angle between the $x$-direction and each point on the annulus. This particular procedure was introduced in Jackiewicz et al. (2007b) and is similar to what is usually done in standard time-distance measurements (Duvall et al., 1997). The temporal cross-covariance functions are computed for 20 different annulus radii ( $\Delta=1.46$ to $29.2 \mathrm{Mm}$, incremented by $\delta \Delta=1.46 \mathrm{Mm}$ ) for each mode ridge and measurement type, which yields a set of functions $C^{\alpha, n}(\mathbf{r}, t ; \Delta)$, where $\alpha=\{$ oi, we, ns $\}$. Flows introduce asymmetries in time lag $[t]$ in the cross-covariance functions.

Travel times are then measured according to the procedure developed by Gizon and Birch (2002, 2004). This method establishes a linear relationship between the travel-time perturbations $[\tau]$ and the cross-covariances, given by

$$
\tau_{n}^{\alpha}(\mathbf{r}, \Delta)=h_{t} \sum_{t} W^{n}(\Delta, t)\left[C^{\alpha, n}(\mathbf{r}, t ; \Delta)-C^{\mathrm{ref}, n}(\mathbf{r}, t ; \Delta)\right],
$$

where the sum over $t$ denotes a discrete sum over the entire observation time [T] (for this work $T=$ one day in most cases), $h_{t}=$ one minute is the temporal sampling rate of the data, $W$ are weight functions, and $C^{\text {ref }}$ is a reference cross-covariance symmetric in time lag, which we choose to be the cross-covariance computed from our model power spectrum. This model is tuned to match the observed power spectrum of each individual ridge (see Section 3). $C^{\text {ref }}$ is the inverse Fourier transform of the model power spectrum. Full details about the $W$ function and Equation (3) can be found in Gizon and Birch (2004). The resulting three different types of travel-time measurements are constructed to have sensitivity to different flow geometries. The "oi" travel times are highly sensitive to horizontal flow divergence, whereas the "we" and "ns" travel times give information about directional flows.

\section{Forward Modeling}

We now briefly discuss the steps that we have taken to model the ridge-filtered travel-time measurements described in the previous section, as well as the measurement noise properties. Full details of forward modeling in time-distance helioseismology can be found in Gizon and Birch (2002, 2004), and Birch and Gizon (2007).

\subsection{Travel-Time Sensitivity Kernels for Ridge Filtering}

We consider travel-time measurements of type $\alpha=\{$ oi, we, ns $\}$ for each distance $[\Delta]$ and for each mode ridge $[n]$ as described in the previous section. The travel-time perturbations are related to the small-amplitude flows through a linear relation:

$$
\tau_{n}^{\alpha}\left(\mathbf{r}_{i} ; \Delta\right)=h_{r}^{2} h_{z} \sum_{j, z} \mathbf{K}^{\alpha, n}\left(\mathbf{r}_{j}-\mathbf{r}_{i}, z ; \Delta\right) \cdot \mathbf{u}\left(\mathbf{r}_{j}, z\right)+\mathcal{N}_{\mathrm{tt}}^{\alpha, n}\left(\mathbf{r}_{i} ; \Delta\right),
$$

where $\mathbf{K}$ denotes the three-dimensional travel-time sensitivity kernel, $\mathbf{u}$ is the real vector flow in the Sun, $\mathcal{N}_{\mathrm{tt}}$ represents the noise in the travel times [tt], and the sum over the vertical 

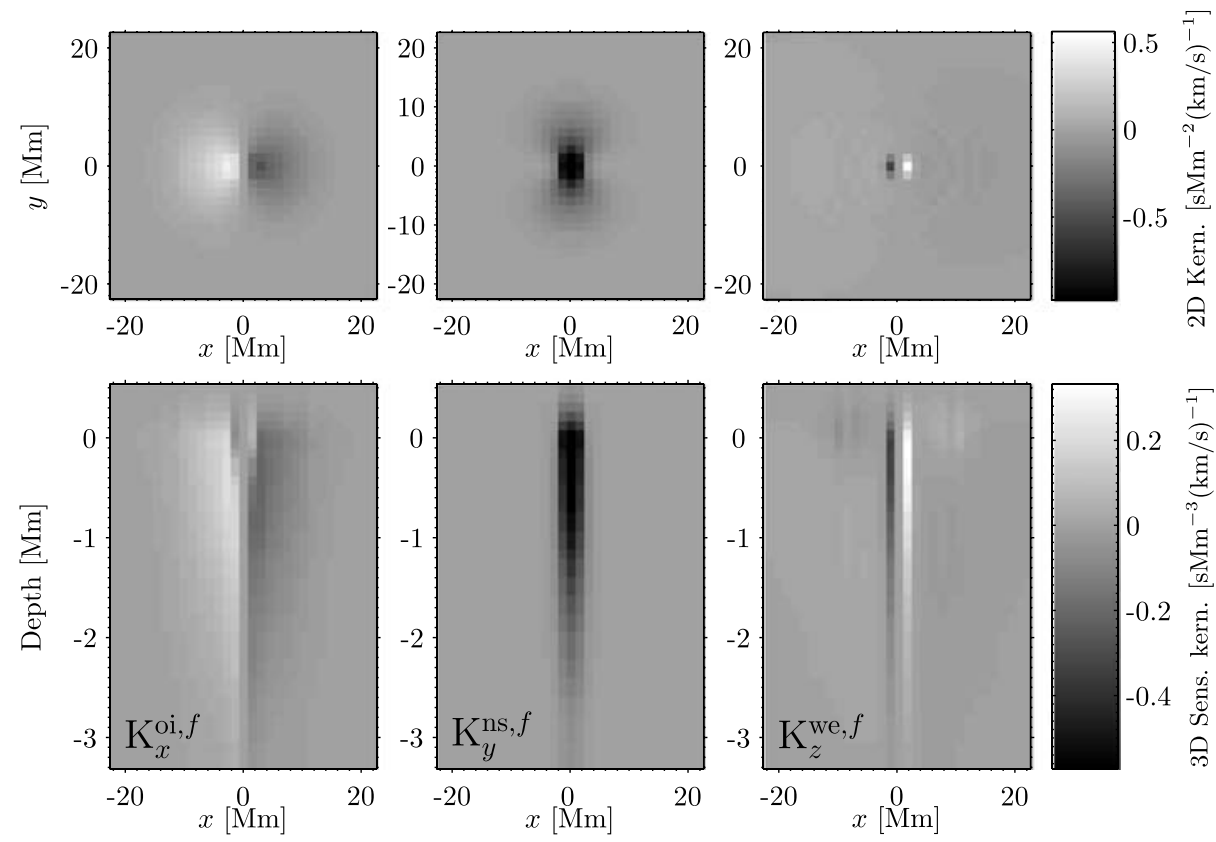

Figure 1 Examples of various sensitivity kernels $\mathbf{K}^{\alpha, f}(\mathbf{r}, z ; \Delta)$ used in this study and defined in Equation (4) obtained by computing weighted azimuthal averages of the point-to-point kernels for the $\alpha$ point-to-annulus geometries. The kernels in the left column give the sensitivity of "oi" $f$-mode travel times to $u_{x}$, and the kernels in the middle (right) column give the sensitivity of the "ns" ("we") $f$-mode travel times to $u_{y}\left(u_{z}\right)$. The top row are 2D kernels obtained after integrating the 3D kernels over depth. The bottom row shows depth slices along $y=0$ of the 3D kernels. The type $[\alpha]$ and mode ridge $[n]$ of the kernels in each column is indicated in the bottom panels. For each case, $\Delta=10.2 \mathrm{Mm}$. The gray scale in the bottom row has been truncated to $75 \%$ of kernel maximum.

coordinate $[z]$ denotes a discrete sum (where we note that $z=0$ at the surface and is negative inside the Sun). The kernels are computed so that the horizontal grid spacing, $h_{x}=h_{y}=$ 1.46 Mm and $h_{r}^{2}=h_{x} h_{y}$, matches that of the travel-time measurements. The vertical grid (with spacing $h_{z}$ ) is taken from the model on which the kernels are computed. The kernel $[\mathbf{K}]$ is computed in the first Born approximation (Gizon and Birch, 2002). We start with the point-to-point kernels for flows derived in Birch and Gizon (2007), computed for the $f, p_{1}, p_{2}, p_{3}$, and $p_{4}$ ridges, whose input power spectra have been tuned to match the ridge-filtered observed power spectra. For each ridge, 20 kernels are computed, one for each $\Delta$. The $3 \mathrm{D}$ point-to-point kernels are then azimuthally averaged according to the three different point-to-annulus weighting geometries $\alpha$ used for the travel-time measurements. The total number of point-to-annulus kernels thus obtained is 300 , with each kernel having three components. A few examples of $f$-mode kernels after weighted azimuthal averaging are shown in Figure 1 for $\mathbf{r}_{i}=0$. From left to right, the columns show kernels that give the sensitivity to $u_{x}, u_{y}$, and $u_{z}$, respectively. The top row shows the resulting $2 \mathrm{D}$ kernel after integration over depth.

As mentioned in the introduction, the motivation behind the inversion we choose to perform is that the ridge-filtered kernels to a very good approximation separate into a horizontal 2D function of $\mathbf{r}$ times a 1D function of depth $z$. In other words, dropping the labels $\alpha, n$, and $\Delta$ for the moment, we can write the $i$ th component of the sensitivity kernel defined in 

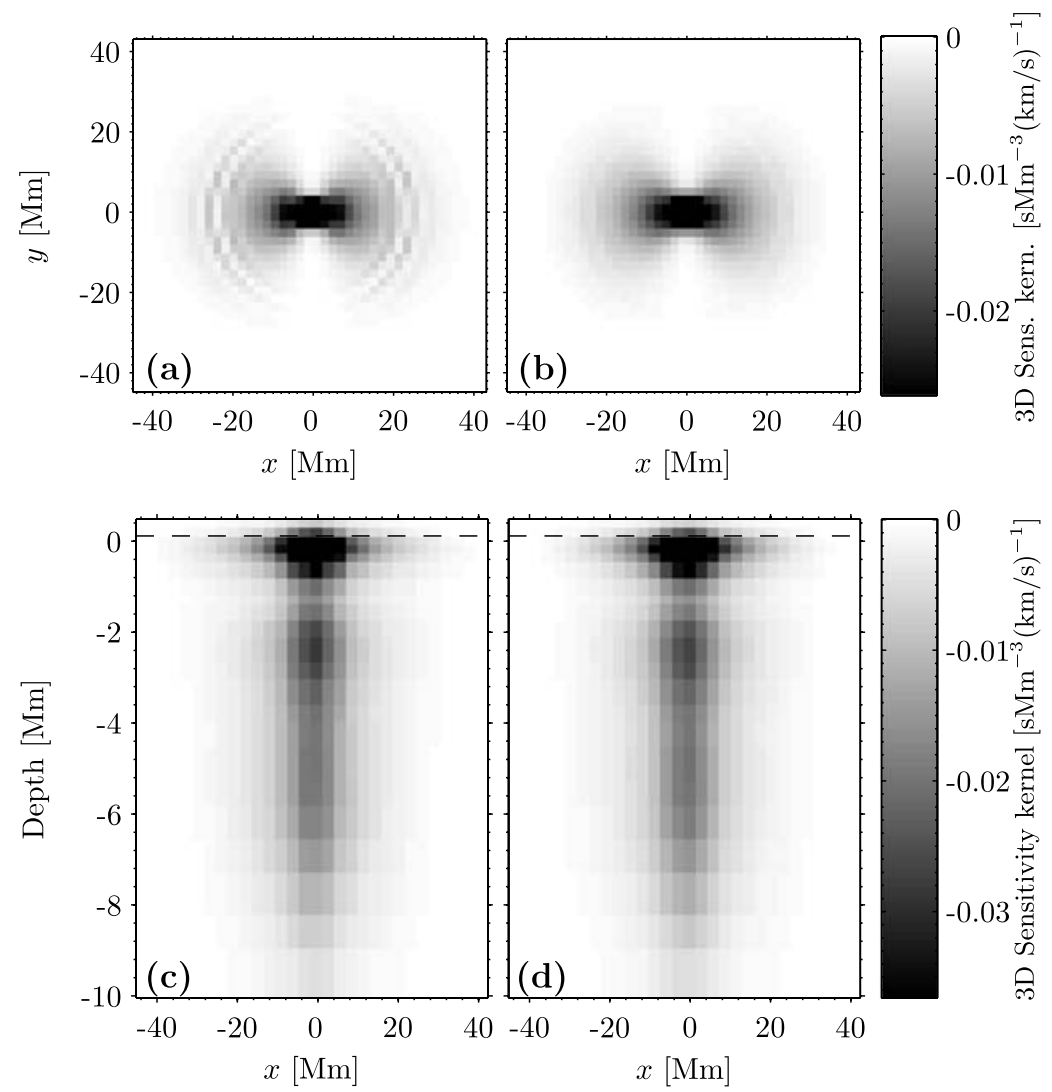

Figure 2 Separability of a sensitivity kernel for $p_{2}$ and for ridge filtering. This particular kernel, $\mathrm{K}_{x}^{\mathrm{we}, p_{2}}$, gives the sensitivity of "we" $p_{2}$-mode travel times to $u_{x}$ for $\Delta=23.4 \mathrm{Mm}$. (a) Horizontal cut through the kernel at a height of about $100 \mathrm{~km}$ above the photosphere, denoted by the dashed line in the panel below. (b) Horizontal cut through the quantity obtained as the product of the two (normalized) functions, $f(\mathbf{r})$ and $g(z)$, computed by integrating the kernel in (a) horizontally and over depth, according to Equations (5) and (6). (c) A depth slice along $y=0$ of the original kernel from (a). (d) A depth slice along $y=0$ of the kernel in (b). The match between the left and right columns is quite good. The gray scale has been truncated to $40 \%$ of the maximum value for ease of comparison.

Equation (4) as the product

$$
\mathrm{K}_{i}(\mathbf{r}, z) \approx f_{i}(\mathbf{r}) g_{i}(z),
$$

where

$$
f_{i}(\mathbf{r})=\frac{h_{z} \sum_{z} \mathrm{~K}_{i}(\mathbf{r}, z)}{\sqrt{h_{r}^{2} h_{z} \sum_{j, z} \mathrm{~K}_{i}\left(\mathbf{r}_{j}, z\right)}}, \quad g_{i}(z)=\frac{h_{r}^{2} \sum_{j} \mathrm{~K}_{i}\left(\mathbf{r}_{j}, z\right)}{\sqrt{h_{r}^{2} h_{z} \sum_{j, z} \mathrm{~K}_{i}\left(\mathbf{r}_{j}, z\right)}} .
$$

In Figure 2 we demonstrate the separability for an example $p_{2}$ and "we"-averaged kernel. Figure 2(a) shows a slice of the $\mathrm{K}_{x}^{\mathrm{we}, \mathrm{p}_{2}} \mathrm{kernel}$ at about $100 \mathrm{~km}$ above the photosphere, and in Figure 2(b) we show the same slice, but of the function obtained by Equation (5). In the bottom two panels, depth slices of these two kernels are shown for comparison. The match between the kernels is quite good. We have also studied the point-to-point kernels in this 
way. In general, the weighted azimuthally averaged kernels (the ones actually used in the inversion) separate "better" than the point-to-point ones, because much of the small-scale structure is averaged away. All of the other kernels for ridge filtering that we have studied separate this way to a very good approximation, giving us confidence in the type of inversion we choose to employ.

\subsection{Noise-Covariance Matrix}

Travel times contain a significant amount of realization noise and a good understanding of these noise properties allows us to assign accurate errors to the flow estimates. It has been shown in previous time-distance inversions that it is important to take into account the noise-covariance matrix (Jensen, Duvall, and Jacobsen, 2003; Couvidat et al., 2005; Couvidat, Birch, and Kosovichev, 2006). Gizon and Birch (2004) showed in detail how to compute model noise covariances of travel times.

We assume that the solar oscillations are stationary and spatially homogeneous since we are restricting our study to the quiet Sun. We further assume that the noise between different ridge measurements $n$ and $n^{\prime}$ is uncorrelated. This approximation is acceptable for this study because we carry out ridge filtering. The covariance matrix $\Lambda$ of the noise components $\mathcal{N}_{\mathrm{tt}}$ from Equation (4) is given by

$$
\Lambda_{n}^{\alpha \beta}\left(\mathbf{r}_{i}-\mathbf{r}_{j} ; \Delta, \Delta^{\prime}\right)=\operatorname{Cov}\left[\mathcal{N}_{\mathrm{tt}}^{\alpha, n}\left(\mathbf{r}_{i} ; \Delta\right), \mathcal{N}_{\mathrm{tt}}^{\beta, n}\left(\mathbf{r}_{j} ; \Delta^{\prime}\right)\right] .
$$

This quantity has units of $s^{2}$ and is computed according to Equation (28) in Gizon and Birch (2004). Example plots for the case when $n=f$ and for $\Delta=5 \mathrm{Mm}$ were shown in Jackiewicz et al. (2007b) using the same model. Similar features are seen for all mode ridges and distances considered here. Note that the covariance matrix elements of $\Lambda$ scale with the observation time $T$ as $T^{-1}$.

\section{Basic Strategy of the 2+ 1 Dimensional Subtractive Optimally Localized Averages Inversion}

The problem we wish to solve is to estimate, for example, $u_{x}(\mathbf{r}, z)$ in Equation (4), given the travel-time measurements, the sensitivity kernels, and the noise-covariance matrix. We carry this out using a $2+1 \mathrm{D}$ SOLA inversion procedure. We formulate the problem in terms of the component $u_{x}$ only for notational simplicity, noting that the procedure for finding all other flow components is completely equivalent. We first discuss the basic idea of this method, and then in the following two sections separately describe the 2D and 1D parts in more detail.

An OLA-type inversion for our purposes seeks a way to combine the sensitivity kernels to find a three-dimensional averaging kernel that is, roughly, spherical, centered horizontally about the origin $\mathbf{r}=0$ and vertically about the target depth $z_{t}$ located somewhere in the solar interior. The averaging kernel will be found from the $2+1 \mathrm{D}$ inversion and is defined by

$$
v_{x}\left(\mathbf{r} ; z_{t}\right)=h_{r}^{2} h_{z} \sum_{j, z}{ }^{2+1 \mathrm{D}} \mathcal{K}^{u_{x}}\left(\mathbf{r}_{j}-\mathbf{r}, z ; z_{t}\right) \cdot \mathbf{u}\left(\mathbf{r}_{j}, z\right)+\text { noise }
$$

where $v_{x}$ is an estimate of the real solar flow $u_{x}$ (as is the case in the rest of the paper) and the noise term will be specifically quantified in the following. To clarify the notation, sensitivity kernels are written as $\mathrm{K}$ and averaging kernels are written as $\mathcal{K}$. Any superscript 
to the left of the averaging kernel denotes by which type of inversion it was computed, for example, ${ }^{1 \mathrm{D}} \mathcal{K},{ }^{2 \mathrm{D}} \mathcal{K}$, and ${ }^{2+1 \mathrm{D}} \mathcal{K}$. The superscripts to the right indicate for which component of $\mathbf{u}$ and ridge $n$ the averaging kernel is computed.

It is important to observe from Equation (8) that the $y$ - and $z$-components of the averaging kernel should be zero, so that the flow estimate $\left[v_{x}\right]$ is not contaminated by any cross-talk from $u_{y}$ and $u_{z}$. As seen in the next section, the $2 \mathrm{D}$ inversion attempts to accomplish this by constraining the spatial integral of $\mathcal{K}_{y}$ and $\mathcal{K}_{z}$ to be zero. Ideally, one would want the $x$-component of the averaging kernel to be a $\delta$ function; however, noise and a finite set of travel times inhibit this. Nonetheless, if an acceptable averaging kernel is found, then the travel times can be properly averaged to give an estimate of the local flow $v_{x} \approx u_{x}$ in which we are interested.

Since the problem essentially separates into a $2 \mathrm{D}$ and a $1 \mathrm{D}$ problem because of ridge filtering, the three-dimensional averaging kernel ${ }^{2+1 \mathrm{D}} \mathcal{K}^{u_{x}}$ from Equation (8) is derived in two main steps. The first step is to compute the $2 \mathrm{D}$ (horizontal) component of the averaging kernel, ${ }^{2 \mathrm{D}} \mathcal{K}^{u_{x}}(\mathbf{r})$, such that its $x$-component is highly peaked about the point $\mathbf{r}=0$, and the other components are zero. This typically involves trying to match the $x$-component to a target function that is a $2 \mathrm{D}$ Gaussian function in horizontal coordinates $\mathbf{r}=(x, y)$. The inversion coefficients, or weights $w$, that accomplish this averaging of the sensitivity kernels, are then used to combine the travel times in such a way that the estimated flow for any ridge measurements $n$ is

$$
v_{x}^{n}(\mathbf{r} ; \Delta)=\sum_{j, \alpha} w^{\alpha, n}\left(\mathbf{r}_{j}-\mathbf{r} ; \Delta\right) \tau_{n}^{\alpha}\left(\mathbf{r}_{j} ; \Delta\right)
$$

The resulting flow map $\left[v_{x}^{n}\right]$ is an average of the real flow $\left[u_{x}\right]$ over the depth that the dominant modes of ridge $n$ probe. An intermediate step is to combine these maps over all distances $[\Delta]$ by an averaging procedure based on the correlated noise in the measurements. A set of weightings $[\gamma]$ is computed, described in detail in Section 5.1, such that the distance-averaged flows are given by

$$
v_{x}^{n}(\mathbf{r})=\sum_{j} \gamma_{j}^{n} v_{x}^{n}\left(\mathbf{r} ; \Delta_{j}\right)
$$

where $\gamma_{j}$ is defined in Equation (21) and the sum $j$ runs over all $\Delta$ used in the problem.

The second main step is to obtain localization of the 3D averaging kernel in the vertical direction about $z_{t}$ by combining separate $2 \mathrm{D}$ ridge measurements. A $1 \mathrm{D}$ inversion in depth is thus performed that seeks a new set of inversion coefficients $c^{n}$. These new coefficients combine the 2D flow maps in Equation (10) in such a way that the final estimate of the flow around $z_{t}$ is given by

$$
v_{x}\left(\mathbf{r} ; z_{t}\right)=\sum_{n} c^{n}\left(z_{t}\right) v_{x}^{n}(\mathbf{r}) \approx u_{x}\left(\mathbf{r} ; z_{t}\right),
$$

where $u_{x}\left(\mathbf{r} ; z_{t}\right)$ denotes the real flow at a particular depth $z_{t}$. The whole procedure is in principle carried out to estimate each flow component $\left(u_{x}, u_{y}, u_{z}\right)$ at many different target depths $\left[z_{t}\right]$ to infer the vector flow $[\mathbf{u}(\mathbf{r}, z)]$ throughout a desired interior region. We now describe in more detail how the 2D and 1D inversion weights are computed in the following two sections. 


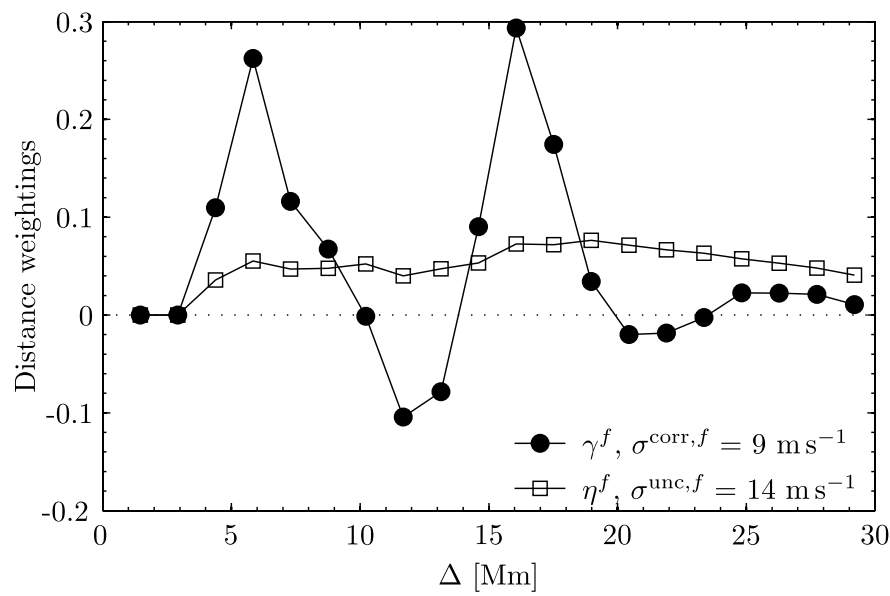

Figure 3 Example distance weightings from a 2D $f$-mode inversion for $u_{x}$ using a target resolution of $11 \mathrm{Mm}$, as described in Section 5.1. The observation time is 24 hours. The open squares are the weights at each distance obtained by assuming that the estimated flows $v_{x}^{f}(\mathbf{r} ; \Delta)$ are uncorrelated for different $\Delta$ (Equation (17)). The filled circles are the weights obtained by taking the correlations properly into account (Equation (21)). The noise $\sigma$ for each case is indicated in the legend.

\section{2D Horizontal Inversion}

Based on the separability of the sensitivity kernels discussed in Section 3, the 2D inversion is formulated to solve Equation (4) for $v_{x}(\mathbf{r})$ by using the 2D depth-integrated kernel

$$
\mathbf{K}^{\alpha, n}(\mathbf{r} ; \Delta)=h_{z} \sum_{z} \mathbf{K}^{\alpha, n}(\mathbf{r}, z ; \Delta),
$$

which we compute for all $\alpha, n$, and $\Delta$ available.

We can define the averaging kernel that we wish to find from the $2 \mathrm{D}$ inversion, ${ }^{2 \mathrm{D}} \mathcal{K}^{u_{x}, n}$, by inserting Equation (4) into Equation (9) to obtain

$$
\begin{aligned}
v_{x}^{n}(\mathbf{r} ; \Delta)= & h_{r}^{2} h_{z} \sum_{i, z}{ }^{2 \mathrm{D}} \mathcal{K}^{u_{x}, n}\left(\mathbf{r}_{i}-\mathbf{r}, z ; \Delta\right) \cdot \mathbf{u}\left(\mathbf{r}_{i}, z\right) \\
& +\sum_{j, \alpha} w^{\alpha, n}\left(\mathbf{r}_{j}-\mathbf{r} ; \Delta\right) \mathcal{N}_{\mathrm{tt}}^{\alpha, n}\left(\mathbf{r}_{j} ; \Delta\right)
\end{aligned}
$$

where

$$
{ }^{2 \mathrm{D}} \mathcal{K}^{u_{x}, n}(\mathbf{r}, z ; \Delta)=\sum_{j, \alpha} w^{\alpha, n}\left(\mathbf{r}_{j} ; \Delta\right) \mathbf{K}^{\alpha, n}\left(\mathbf{r}-\mathbf{r}_{j}, z ; \Delta\right) .
$$

This shows explicitly that the averaging kernel gives only an estimate $v_{x}^{n}$ of the flow that is an average of the real flows over some depth.

The details for obtaining the inversion weights $w$ for a 2D SOLA inversion for flows were presented in Jackiewicz et al. (2007b); however, in that work only one mode ridge, one distance $\Delta$, and two components of the sensitivity kernels $\left(\mathrm{K}_{x}\right.$ and $\left.\mathrm{K}_{y}\right)$ were utilized. The generalization to our present case is straightforward. To summarize this procedure, we first prescribe a target function ${ }^{2 \mathrm{D}} \mathcal{T}^{u_{x}}$ that we wish the averaging kernel to resemble. It is 
a vector-valued function, chosen such that the $x$-component is typically a 2D Gaussian in $\mathbf{r}$ with dispersion $\sigma$, and the other components are zero:

$$
{ }^{2 \mathrm{D}} \mathcal{T}^{u_{x}}(\mathbf{r})=\left(\frac{\mathrm{e}^{-r^{2} / 2 \sigma^{2}}}{2 \pi \sigma^{2}}, 0,0\right),
$$

where $r=\|\mathbf{r}\|$ is the 2D vector norm. The horizontal integral of the target function is normalized to one. The full width at half-maximum (FWHM $=2 \sigma \sqrt{2 \ln 2}$ ) of the target function is a measure of the resolution of the inversion if the averaging kernel matches it well. For the sake of completeness, in an inversion for the $j$ th component of $\mathbf{u}$, with the Gaussian function in Equation (15) denoted as $G(r)$, the $i$ th component of the target function is ${ }^{2 \mathrm{D}} \mathcal{T}_{i}^{u_{j}}=G(r) \hat{\mathbf{e}}_{i} \delta_{i j}$, where $\hat{\mathbf{e}}_{i}$ is the unit vector in the $i$ th direction and $\delta$ is the Kronecker delta function.

Two quantities, one that measures the mismatch between the averaging kernel and target function and another that measures the noise propagation, are computed. Let the noise in the inversion for $u_{x}$ for ridge $n$ be denoted by $\mathcal{N}^{u_{x}, n}$; it is specifically defined in Section 5.1. A minimization (with respect to the inversion weights) is carried out according to

$$
\min _{w} \sum_{i}\left\|^{2 \mathrm{D}} \mathcal{K}^{u_{x}, n}\left(\mathbf{r}_{i}\right)-{ }^{2 \mathrm{D}} \mathcal{T}^{u_{x}}\left(\mathbf{r}_{i}\right)\right\|^{2}+\beta\left(\mathcal{N}^{u_{x}, n}\right)^{2},
$$

where $\beta$ is some regularization parameter that we choose typically to be quite small (Jackiewicz et al., 2007b). A large matrix is then regularized and inverted for each value of the trade-off parameter, which results in a unique set of weights at each point in this parameter space. We choose an "optimal" set of weights $[w]$ from examining the trade-off curve (or "L" curve) as discussed in Jackiewicz et al. (2007b), such that the averaging kernel matches closely the target function. The $2 \mathrm{D}$ averaging kernel is constructed by convolution of the sensitivity kernels with the weights according to Equation (14).

Since the inversion in this example is carried out for $u_{x}^{n}$, it is important that ${ }^{2 \mathrm{D}} \mathcal{K}_{y}^{u_{x}, n}$ and ${ }^{2 \mathrm{D}} \mathcal{K}_{z}^{u_{x}, n}$ be as close to zero as possible, to minimize the "cross-talk" among all of the components. This is achieved in practice by constraining the total spatial integrals of the $y$ and $z$-components to be zero, although in practice there is usually some structure present even with this constraint. We are in the process of exploring other effective constraints. When a well-localized averaging kernel is found for each ridge $[n]$, the weights are then suitable to be used to average the travel times to give an estimate of the flow $v_{x}^{n}(\mathbf{r})$ using Equation (9).

\subsection{Combining All of the Distances}

Throughout this inversion procedure, it is necessary to combine the quantities we obtain for different annulus radii $[\Delta]$, such as the estimated flow maps $v_{x}^{n}(\mathbf{r} ; \Delta)$. This is accomplished by weighting each distance appropriately. One simple way of achieving this, typically used in helioseismology, is to assume that the noise in each measurement is independent and uncorrelated. Then the standard deviation in the estimated flow maps is used to determine the contribution of the errors at each distance. We denote the standard deviation of a set of flows $\left[v_{x}^{n}\right]$ for each distance $\left[\Delta_{j}\right]$ and mode ridge $[n]$ as $\sigma_{j}^{\text {unc, } n}$. The "unc" superscript emphasizes the assumption of uncorrelated data. Finding the minimum variance of this set then gives weighting factors $\eta_{j}^{n}$ according to

$$
\eta_{j}^{n}=\frac{\left(1 / \sigma_{j}^{\mathrm{unc}, n}\right)^{2}}{\sum_{j=1}^{20}\left(1 / \sigma_{j}^{\mathrm{unc}, n}\right)^{2}},
$$


where the sum in the denominator runs over all 20 distances used in this problem. We carry out a $2 \mathrm{D}$ inversion as described earlier for $u_{x}$ using $f$ modes in a region of quiet Sun (the same region used in Section 8). The weights obtained from the estimated flows using Equation (17) are plotted in Figure 3 as open squares. For very small distances, where the noise is very high, the weights are nearly zero. From the total variance we obtain a noise estimation given by

$$
\sigma^{\mathrm{unc}, \mathrm{f}}=\left[\sum_{j}\left(1 / \sigma_{j}^{\mathrm{unc}, \mathrm{f}}\right)^{2}\right]^{-1 / 2},
$$

which for this particular example is $14 \mathrm{~m} \mathrm{~s}^{-1}$.

However, we know that the values of the flows at different $\Delta$ are correlated quite strongly due to noise (Gizon and Birch, 2004), and so we choose to average them in a way that takes these correlations into account. A covariance matrix $C_{n}$ of the noise in the individual flow measurements at distances $\Delta$ and $\Delta^{\prime}$ of ridge $n$ is computed by using the $2 \mathrm{D}$ inversion weights as

$$
C_{n}\left(\Delta, \Delta^{\prime}\right)=\sum_{i, j, \alpha, \beta} w^{\alpha, n}\left(\mathbf{r}_{i} ; \Delta\right) \Lambda_{n}^{\alpha \beta}\left(\mathbf{r}_{i}-\mathbf{r}_{j} ; \Delta, \Delta^{\prime}\right) w^{\beta, n}\left(\mathbf{r}_{j} ; \Delta^{\prime}\right),
$$

where $\Lambda$ is the covariance matrix of the noise in the travel times (Equation (7)) and $w$ are the 2D inversion weights. Note that the matrix $C$ has units of length ${ }^{2}$ time $^{-2}$. The final measurement of any general quantity $[q]$ for each mode ridge is then obtained by averaging the 20 distances we use according to (for example, see Schmelling, 1995)

$$
q^{n}(\mathbf{r})=\sum_{j=1}^{20} \gamma_{j}^{n} q^{n}\left(\mathbf{r} ; \Delta_{j}\right)
$$

where the set of weightings $\left[\gamma_{j}^{n}\right]$ is given by

$$
\gamma_{j}^{n}=\frac{\left(\sum_{i=1}^{20} C_{n}^{-1}\left(\Delta_{i}, \Delta_{j}\right)\right)}{\left(1 / \sigma^{\operatorname{corr}, n}\right)^{2}}
$$

and the variance $\left(\sigma^{\text {corr }, n}\right)^{2}$ for the correlated case is

$$
\left(\sigma^{\mathrm{corr}, n}\right)^{2}=\left(\sum_{i j}\left(C_{n}^{-1}\left(\Delta_{i}, \Delta_{j}\right)\right)\right)^{-1}
$$

We can now identify $\mathcal{N}^{u_{x}, n} \equiv \sigma^{\text {corr, } n}$, the noise in a measurement of $v_{x}^{n}$, introduced in Equation (16). A set of weights for the $f$-mode case $\left[\gamma_{j}^{f}\right]$ obtained this way is plotted as the filled circles in Figure 3 to compare with the uncorrelated case. What is interesting to note is that for some distances a negative contribution is needed to average the data properly, which is never the case for the uncorrelated data. The estimated noise is also lower than in the uncorrelated case. Equation (20) is quite general and has been used to obtain the distance-averaged flow maps presented in Section 8, as well as all of the averaging kernels shown in this paper. Quantities written without the distance argument $\Delta$ have been averaged according to Equation (20). 


\subsection{Averaging Kernels from the $2 \mathrm{D}$ Inversion}

Example averaging kernels after combining all distances for a 2D inversion are shown in Figures $4-7$. In all figures, the top row shows the 2D averaging kernel obtained by integrating over depth the adjacent $3 \mathrm{D}$ averaging kernel in the bottom row. The bottom panels show depth slices along $y=0$. The inherent noise from the inversion corresponding to each figure is given in Table 1.

Figures 4 and 5 show averaging kernels for a 2D inversion for $u_{x}$ for the $f$-and $p_{1}$-mode ridges, respectively, and Figures 6 and 7 are for an inversion for $u_{z}$. The absence of any dominant cross-talk is evident in the top panels of all figures, which are the $2 \mathrm{D}$ averaging kernels that come straight out of the $2 \mathrm{D}$ inversion. There is a completely negligible $y$-component in all cases for the inversions for $u_{x}$. The cross-talk is slightly more pronounced for the $x$ and $y$-components of the kernels in the inversions for $u_{z}$, but it is confined to the very near surface region, typically above the depths that are significant for our inversion results. In general, these averaging kernels are quite good; the only structure from the "off-diagonal" terms are of the order of about $5 \%$ of the diagonal terms. It is important to study averaging kernels such as these to have an idea of what the inversion is actually accomplishing in terms of sensitivity. Similar plots have been examined for all of the other mode ridges available and they exhibit similar features.

\subsection{Minimum Variance}

The averaging kernels from the $2 \mathrm{D}$ inversion in Figures 4-7 are computed for each separate mode ridge $[n]$. One possible way to combine them over all ridges is to use a simple minimum-variance treatment of the noise in the estimated flow component $v_{x}^{n}$, as described in Section 5.1 for the case of distance averaging. Because of ridge filtering, we consider the noise between mode ridges $n$ and $n^{\prime}$ to be uncorrelated. Thus, any quantity $\left[q^{n}\right]$ that depends

Table 1 Values of the relevant quantities for some of the figures in this paper. Listed is the figure number, the type of inversion used to produce the figure, the 1D inversion weights $c^{n}$ (or minimum variance weights $d^{n}$ ), and the estimated noise associated with each measurement ( $1 \sigma$ values). Note that the $1 \mathrm{D}$ inversion weights do not apply for the 2D inversion.

\begin{tabular}{llllllll}
\hline Figure & Inversion type & $c^{f}$ & $c^{p_{1}}$ & $c^{p_{2}}$ & $c^{p_{3}}$ & $c^{p_{4}}$ & ${\text { Noise }\left(\mathrm{m} \mathrm{s}^{-1}\right)}^{2}$ \\
\hline 4 & 2D & - & - & - & - & - & 16 \\
5 & 2D & - & - & - & - & - & 26 \\
6 & 2D & - & - & - & - & - & 17 \\
7 & 2D & - & - & - & - & - & 24 \\
8 & 2D (min. var.) & 0.65 & 0.24 & 0.086 & 0.014 & 0.002 & 21 \\
12 & $2+1 \mathrm{D}$ & 1.02 & -0.04 & -0.01 & $10^{-4}$ & $10^{-4}$ & 27 \\
13 & $2+1 \mathrm{D}$ & -1.2 & 2.2 & $10^{-4}$ & -0.04 & $10^{-4}$ & 40 \\
14 & $2+1 \mathrm{D}$ & -0.17 & -1.7 & 2.3 & 0.5 & 0.05 & 57 \\
18 (a) & $2+1 \mathrm{D}$ & 0 & 1 & 0 & 0 & 0 & 19 \\
18 (b) & $2+1 \mathrm{D}$ & 0.33 & - & 0.47 & 0.17 & 0.05 & 17 \\
20 (top) & $2+1 \mathrm{D}$ & 1.08 & -0.13 & 0.004 & 0.01 & 0.005 & 10 \\
20 (middle) & $2+1 \mathrm{D}$ & -0.11 & 1.01 & 0.03 & 0.04 & 0.01 & 12 \\
20 (bottom) & 2+1D & -0.92 & 1.26 & 0.63 & 0.02 & 0.01 & 22 \\
\hline
\end{tabular}



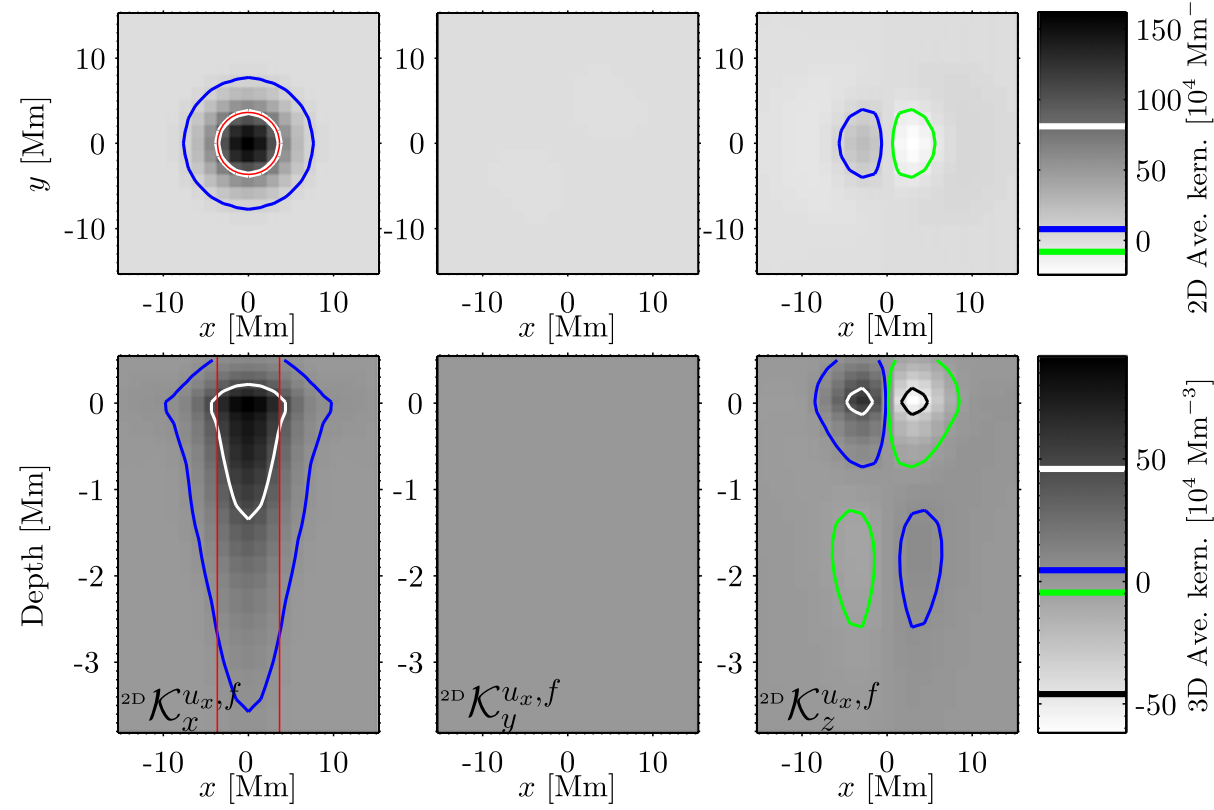

Figure 4 Two-dimensional (top panels) and three-dimensional (bottom panels) averaging kernels for a 2D inversion for $u_{x}$ using only $f$ modes. The panels in the top row show the integral over depth of the corresponding 3D kernel below it. The bottom row shows depth slices of the three components of the 3D kernel along the $y=0$ line. The red line outlines the FWHM $=7.3 \mathrm{Mm}$ of the $2 \mathrm{D}$ Gaussian target function used in the inversion (see Equation (15)). The overplotted color contours, which are also marked on the color bar for reference, denote the following: half-maximum of the kernel (white), negative of the half-maximum (black), and $\pm 5 \%$ of the maximum value of the kernel (blue and green, respectively). Estimates of the noise from this and all other inversions are given in Table 1.

on the set of mode ridges can be averaged according to

$$
q^{u_{x}}=\sum_{n} d^{n} q^{u_{x}, n}
$$

where the weights $d^{n}$ are given by

$$
d^{n}=\frac{\left(1 / \sigma^{\mathrm{corr}, n}\right)^{2}}{\sum_{n}\left(1 / \sigma^{\mathrm{corr}, n}\right)^{2}},
$$

and $\sigma^{\text {corr, } n}$, the correlated noise estimated from the $v_{x}^{n}$ measurements, is defined in Equation (22). In Figure 8 we show the three components of an averaging kernel $\mathcal{K}^{u_{x}}$ obtained by combining five kernels $\mathcal{K}^{u_{x}, n}$ from the minimum variance in $v_{x}^{n}$ using the weights given in Equation (24). The minimum-variance weights for each ridge for this figure are given in Table 1. The noise level is low and the kernel is well localized horizontally; however, there is clearly have no control over the depth at which one wishes to have sensitivity.

In conclusion, the 2D inversion produces averaging functions (such as those shown in Figures 4-8) that are "optimally" localized in the horizontal direction, but not in depth. This depth localization is accomplished by a 1D inversion, to which we now turn. 

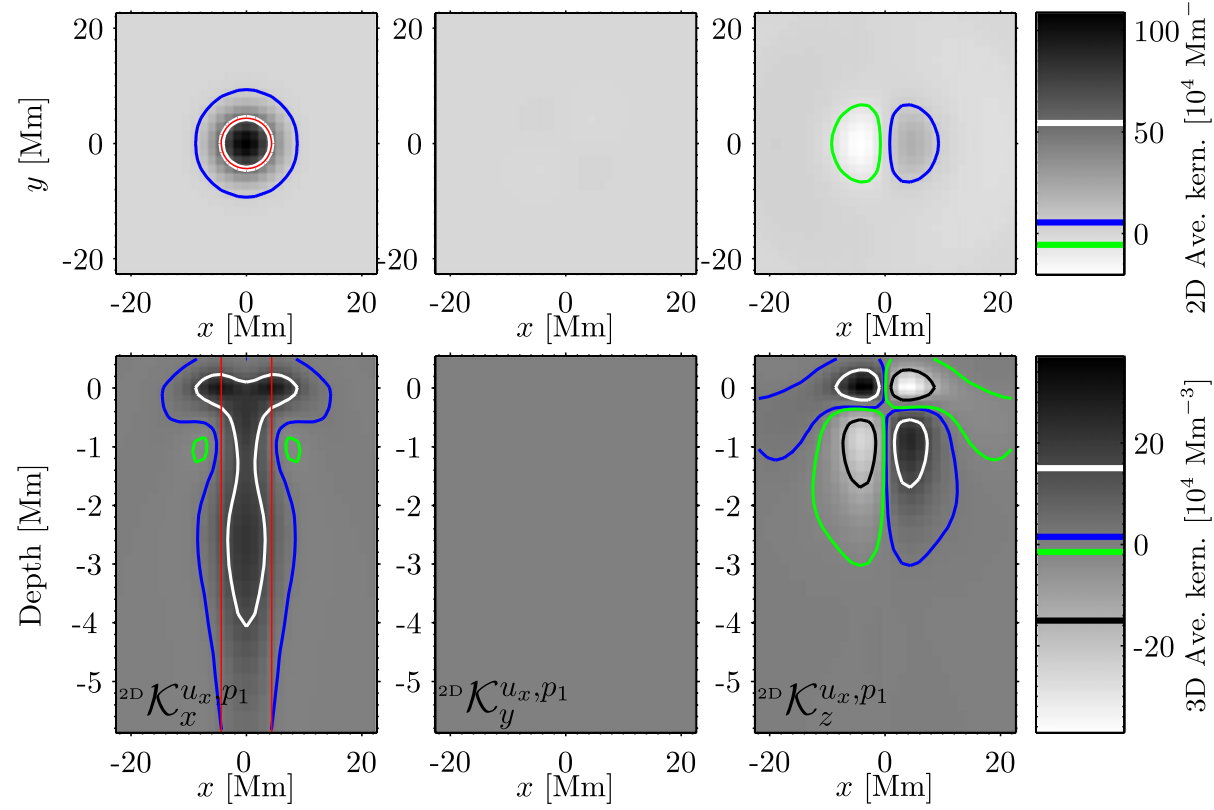

Figure 5 Two-dimensional (top panels) and three-dimensional (bottom panels) averaging kernels for a 2D inversion for $u_{x}$ using only $p_{1}$ modes. The panels in the top row show the integral over depth of the corresponding 3D kernel below it. The bottom row shows depth slices of the three components of the 3D kernel along the $y=0$ line. The red line outlines the FWHM $=7.3 \mathrm{Mm}$ of the $2 \mathrm{D}$ Gaussian target function used in the inversion. The overplotted color contours, which are also marked on the color bar for reference, denote the following: half-maximum of the kernel (white), negative of the half-maximum (black), and $\pm 5 \%$ of the maximum value of the kernel (blue and green, respectively).

\section{1D SOLA Depth Inversion}

Up to this point, the $2 \mathrm{D}$ inversion has provided averaging kernels that average the real solar flows to give estimates for each particular ridge, according to Equation (13). If we assume that the real flows vary slowly in $\mathbf{r}$ over the horizontal extent of the averaging kernel, we can perform the summation in Equation (13) over $\mathbf{r}_{i}$ to obtain

$$
v_{x}^{n}(\mathbf{r}) \approx h_{z} \sum_{z}{ }^{1 \mathrm{D}} \mathrm{K}_{x}^{u_{x}, n}(z) u_{x}(z)+\sum_{j, \alpha} w^{\alpha, n}\left(\mathbf{r}_{j}-\mathbf{r}\right) \mathcal{N}_{\mathrm{tt}}^{\alpha, n}\left(\mathbf{r}_{j}\right)
$$

where

$$
{ }^{1 \mathrm{D}} \mathbf{K}_{x}^{u_{x}, n}(z) \equiv h_{r}^{2} \sum_{i}{ }^{2 \mathrm{D}} \mathcal{K}_{x}^{u_{x}, n}\left(\mathbf{r}_{i}, z\right)
$$

is the $1 \mathrm{D}$ sensitivity kernel for an inversion for $u_{x}$ and mode ridge $n$. Recall that the horizontal integrals of ${ }^{2 \mathrm{D}} \mathcal{K}_{y}$ and ${ }^{2 \mathrm{D}} \mathcal{K}_{z}$ are zero owing to the constraint imposed in the $2 \mathrm{D}$ inversion; therefore, only the $x$-component of the quantities remains in the right-hand sides of Equations (25) and (26). 

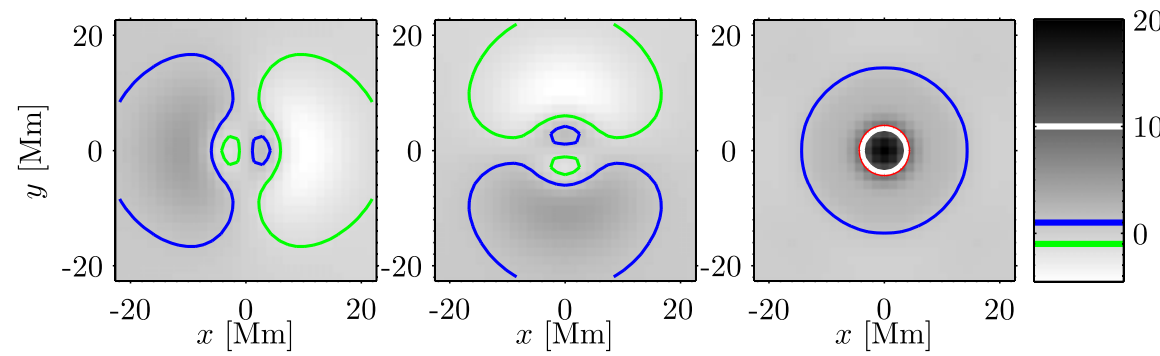

I
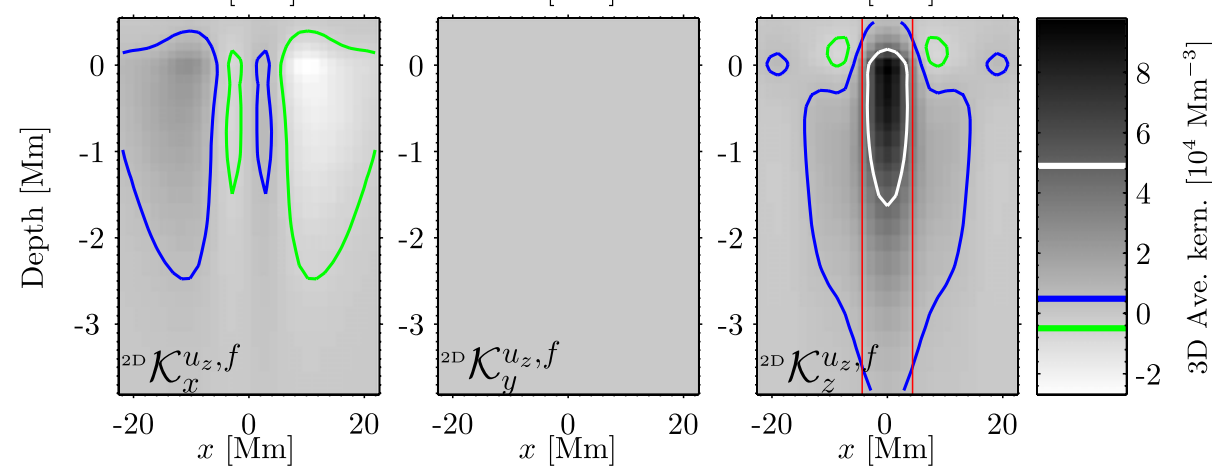

Figure 6 Two-dimensional (top panels) and depth slices of three-dimensional (bottom panels) averaging kernels for a 2D inversion for $u_{z}$ using only $f$ modes. The red line outlines the FWHM $=7.3 \mathrm{Mm}$ of the 2D Gaussian target function used in the inversion. The overplotted color contours, which are also marked on the color bar for reference, denote the following: half-maximum of the kernel (white), negative of the half-maximum (black), and $\pm 5 \%$ of the maximum value of the kernel (blue and green, respectively).

The five available 1D sensitivity kernels are shown in Figure 9. The 1D SOLA inversion seeks inversion coefficients $\left[c^{n}\left(z_{t}\right)\right]$ that combine each ridge measurement about target depth $z_{t}$, so that the final estimate of the flow using Equation (25) is

$$
\begin{aligned}
v_{x}\left(\mathbf{r} ; z_{t}\right) & =\sum_{n} c^{n}\left(z_{t}\right) v_{x}^{n}(\mathbf{r}) \\
& =h_{z} \sum_{z}{ }^{1 \mathrm{D}} \mathcal{K}_{x}^{u_{x}}\left(z ; z_{t}\right) u_{x}(\mathbf{r}, z)+\sum_{j, \alpha, n} c^{n}\left(z_{t}\right) w^{\alpha, n}\left(\mathbf{r}_{j}-\mathbf{r}\right) \mathcal{N}_{\mathrm{tt}}^{\alpha, n}\left(\mathbf{r}_{j}\right),
\end{aligned}
$$

where

$$
{ }^{1 \mathrm{D}} \mathcal{K}_{x}^{u_{x}}\left(z ; z_{t}\right)=\sum_{n} c^{n}\left(z_{t}\right)^{1 \mathrm{D}} \mathrm{K}_{x}^{u_{x}, n}(z)
$$

is the $1 \mathrm{D}$ averaging kernel peaked about $z_{t}$. The $1 \mathrm{D}$ coefficients $\left[c^{n}\right]$ are obtained in an analogous way to the $2 \mathrm{D}$ case. A target function is chosen that is typically a 1D Gaussian in depth, centered about $z_{t}$ :

$$
{ }^{1 \mathrm{D}} \mathcal{T}_{x}^{u_{x}}\left(z ; z_{t}\right)=\frac{\mathrm{e}^{-\left(z-z_{t}\right)^{2} / 2 \sigma^{2}}}{\sigma \sqrt{2 \pi}}
$$



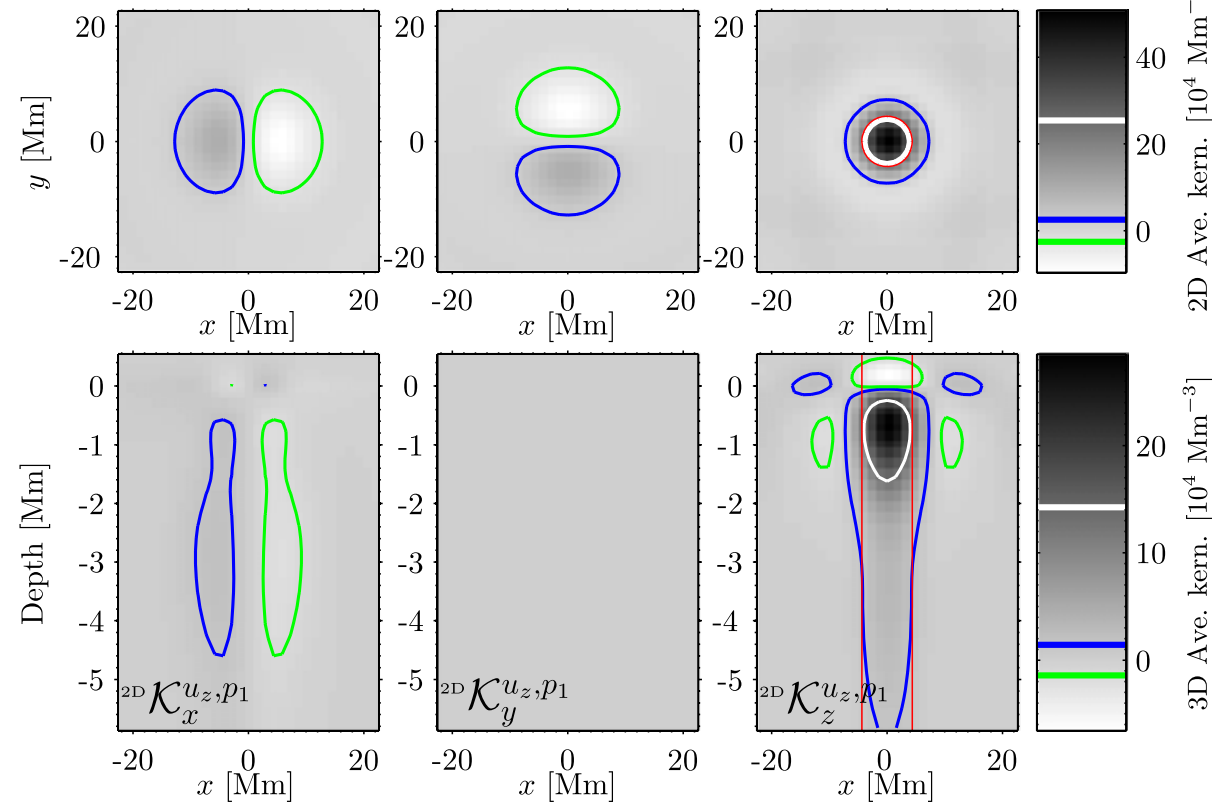

Figure 7 Two-dimensional (top panels) and depth slices of three-dimensional (bottom panels) averaging kernels for a $2 \mathrm{D}$ inversion for $u_{z}$ using only $p_{1}$ modes. The red line outlines the FWHM $=7.3 \mathrm{Mm}$ of the 2D Gaussian target function used in the inversion. The overplotted color contours, which are also marked on the color bar for reference, denote the following: half-maximum of the kernel (white), negative of the half-maximum (black), and $\pm 5 \%$ of the maximum value of the kernel (blue and green, respectively).

A misfit quantity is constructed that measures the mismatch between the target and averaging functions:

$$
\operatorname{misfit}^{2}=h_{z} \sum_{z}\left[{ }^{1 \mathrm{D}} \mathcal{K}_{x}^{u_{x}}\left(z ; z_{t}\right)-{ }^{1 \mathrm{D}} \mathcal{T}_{x}^{u_{x}}\left(z ; z_{t}\right)\right]^{2}
$$

In addition, a parameter that quantifies the error (noise) is included:

$$
\operatorname{error}^{2}=\sum_{n}\left(c^{n} \mathcal{N}^{u_{x}, n}\right)^{2}
$$

A regularization parameter $[\mu]$ is introduced, and a minimization procedure with respect to the weights $\left[c^{n}\right]$ is carried out as

$$
\min _{c}\left[\text { misfit }^{2}+\mu \text { error }^{2}\right]
$$

Computing the minimization results in a system of linear equations, which is solved by inverting a small matrix to obtain the coefficients for each value of the regularization parameter. Finally, we choose weights roughly in the "elbow" of the trade-off curve upon visual inspection. Several example trade-off curves from this procedure are shown in Figure 10 for two target depths $\left[z_{t}\right]$ and different target widths.

In Figure 11 we provide examples of 1D averaging kernels for an inversion for $u_{x}$ and for different target depths. Because of a limited mode set, there is a limited number of depths 

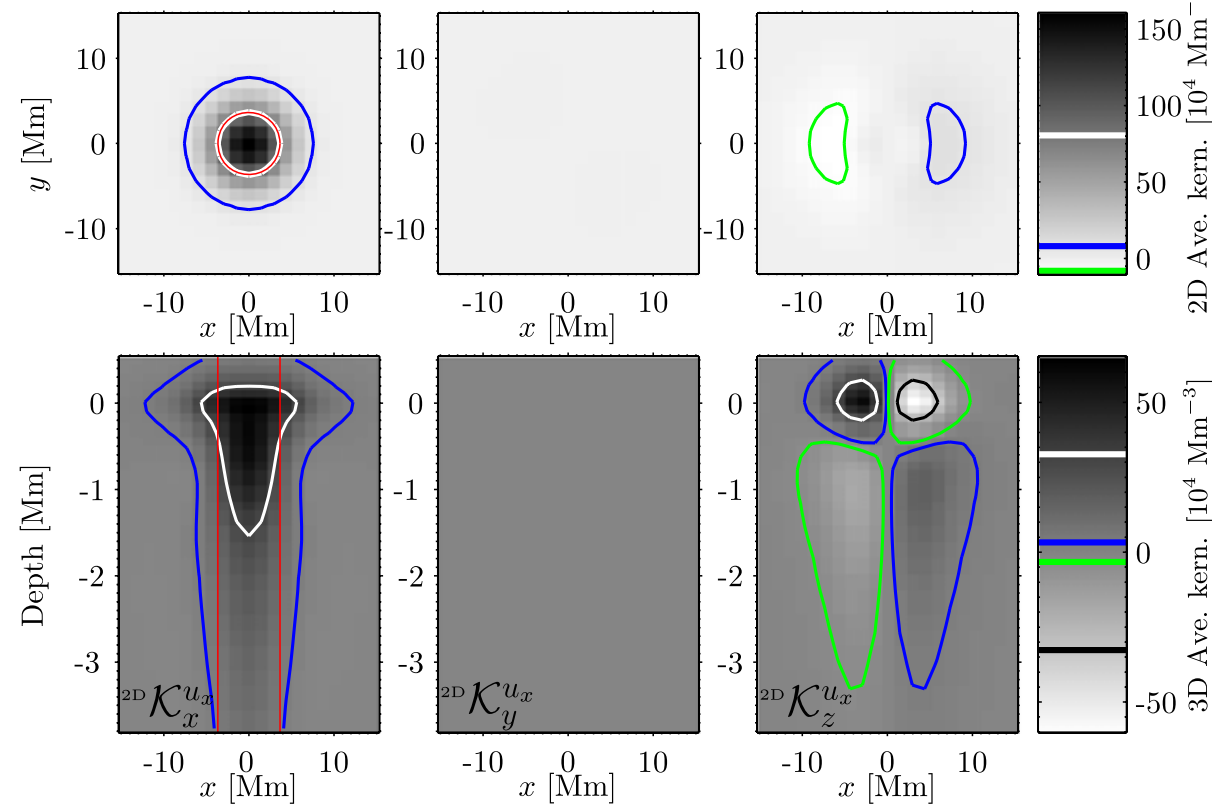

Figure 8 Three-dimensional minimum-variance averaging kernel for a 2D inversion for $u_{x}$. The top panels are the $2 \mathrm{D}$ averaging kernels obtained after integrating the $3 \mathrm{D}$ kernel over depth, and the bottom panels are slices at $y=0$ through the 3D kernel. The method to obtain this kernel is discussed in Section 5.3. The resolution of the inversion is $7.3 \mathrm{Mm}$ and is denoted by the red lines. The overplotted color contours, which are also marked on the color bar for reference, denote the following: half-maximum of the kernel (white), negative of the half-maximum (black), and $\pm 5 \%$ of the maximum value of the kernel (blue and green, respectively).

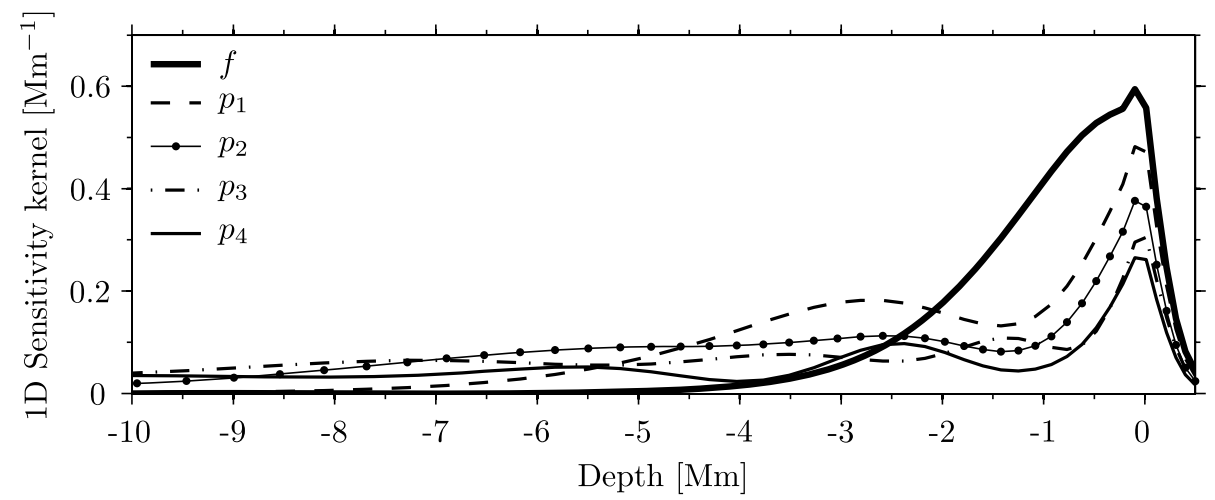

Figure 9 One-dimensional sensitivity kernels $\left[{ }^{1 D} \mathrm{~K}_{x}^{u_{x}, n}(z)\right]$ as a function of depth for each ridge [n] (indicated in the legend) for an inversion for $u_{x}$. These are obtained according to Equation (26).

that can be targeted properly. There is also an obvious limit on the maximum depth with which we can probe with these modes. In addition, as with other helioseismology inversions, a surface component is present (see, e.g., Basu, Antia, and Tripathy, 1999). 


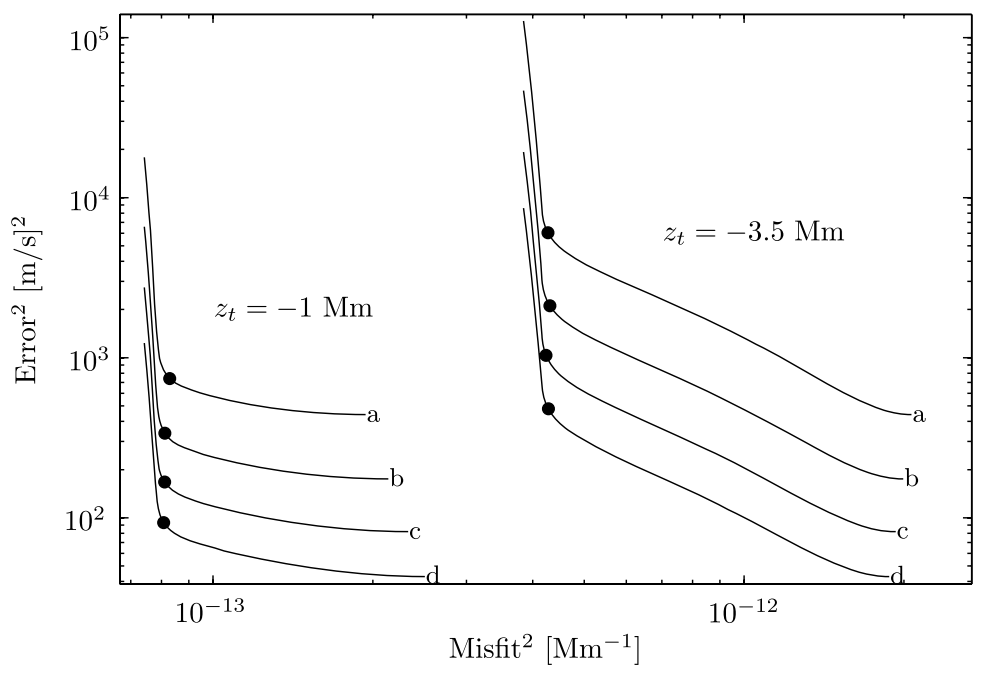

Figure 10 Example trade-off curves computed in the 1D inversion for two target depths. The individual curves in each set of depths are obtained by varying the horizontal target-function width. For the $z_{t}=-1 \mathrm{Mm}$ set, curves a, b, c, and d correspond to widths of 5.8, 7.3, 8.7, and 10.2 Mm, respectively. For the $z_{t}=-3.5 \mathrm{Mm}$ set, a, b, c, and d correspond to 7.3, 8.7, 10.2, and 11.6 Mm, respectively. In this scheme, sets of inversion weights would be chosen at the points given by the filled circles.

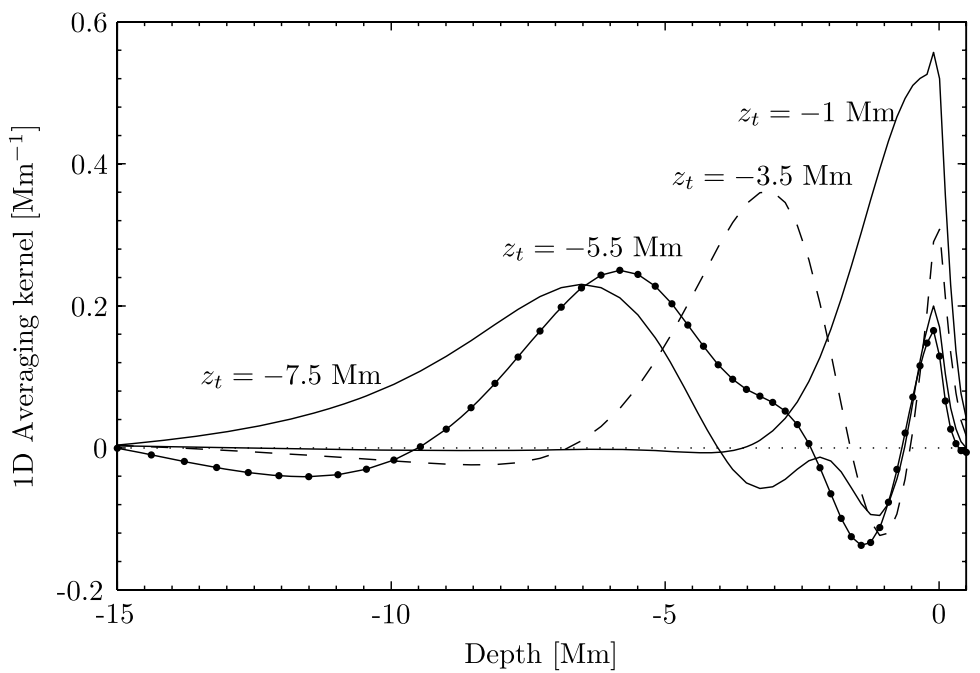

Figure $111 \mathrm{D}$ averaging kernels ${ }^{1 \mathrm{D}} \mathcal{K}^{u_{x}}\left(z ; z_{t}\right)$ for an inversion for $u_{x}$ for different target depths. These are obtained according to Equation (27).

\section{3D Averaging Kernels from the $2+1 D$ Inversion}

We denote the final 3D averaging kernel produced from the $2+1 \mathrm{D}$ inversion for $u_{x}$ as ${ }^{2+1 D} \mathcal{K}^{u_{x}}$. It has been defined in Equation (8) and can be constructed from both sets of inversion coefficients $(w, c)$ in terms of the original sensitivity kernels by using Equations (11) 
and (13):

$$
\begin{aligned}
{ }^{2+1 \mathrm{D}} \mathcal{K}^{u_{x}}\left(\mathbf{r}, z ; z_{t}\right) & :=\sum_{n} c^{n}\left(z_{t}\right)^{2 \mathrm{D}} \mathcal{K}^{u_{x}, n}(\mathbf{r}, z) \\
& :=\sum_{i j \alpha n} c^{n}\left(z_{t}\right) \gamma_{i}^{n} w^{\alpha, n}\left(\mathbf{r}_{j} ; \Delta_{i}\right) \mathbf{K}^{\alpha, n}\left(\mathbf{r}-\mathbf{r}_{j}, z ; \Delta_{i}\right),
\end{aligned}
$$

where we emphasize that the weights $w$ and $c$ are obtained from a specific inversion for $u_{x}$. Recall that the weights $\gamma$ are used to average the quantities over distance $\Delta$.

We now check to see whether the final averaging kernels are as well localized as can be expected from the mode set used, which also justifies separating the problem into 2D and 1D parts. Performing the full $2+1 \mathrm{D}$ inversion for $u_{x}$ for different target depths produces example 3D averaging kernels such as those shown in Figures 12, 13, and 14. Plotted in the left and center panels in each case are depth slices along the $y=0$ and $x=0$ lines of the $x$-component of the kernel, ${ }^{2+1 \mathrm{D}} \mathcal{K}_{x}^{u_{x}}$. The contour of the half-maximum value of the 3D target function is overplotted on the depth slices in red. The white contours show the half-maximum value of the 3D averaging kernel. Note that, in principle, in a noiseless inversion with a large set of available modes, the white contours would perfectly match the red contours. The blue and green contour lines denote $\pm 5 \%$ of the maximum value. The 1D target and averaging functions are provided in the rightmost panel in each figure for comparison. For the shallowest target depth, instead of a simple 1D Gaussian we use a target function that goes to zero at $z=0$. The 1D inversion coefficients $c^{n}$ used to construct the kernels in Figures 12-14 are provided in Table 1. The shallowest depth we can reach with these modes is about $-1 \mathrm{Mm}$, and the deepest is about $-8 \mathrm{Mm}$. Of course, the noise begins to increase quickly with depth.

\section{Results with MDI Data for Quiet-Sun Flows}

In the rest of the paper we provide example flow maps in the quiet Sun from the 2D and $2+1 \mathrm{D}$ inversion procedure discussed here. It is our main intention to demonstrate that the results obtained are sensible and consistent with what might be expected given the spatial resolution, observation time, and level of estimated noise. We note also that to study local flows, we typically remove a mean, large-scale, time-averaged flow from each retrieved map.

\subsection{Tests of the 2D Inversion}

The first simple test we perform is to compare the inferred flows from the $2 \mathrm{D}$ inversion to the direct MDI Doppler data. To accomplish this, the three components of the inferred 2D vector flows are projected onto the line-of-sight vector at each pixel. We use 24 hours of data from the seventh day (26 January 2002) of the nine-day data set available. There is a sunspot in the center of the map that is located vertically about the equator and at $30^{\circ}$ toward the western solar limb for this day. Figure 15 shows the comparison with the Doppler map from a $2 \mathrm{D}$ inversion using only $f$ modes. The correlation between the inferred flows and the Doppler flows is 0.9 for pixels with less than $|10| \mathrm{G}$ of magnetic field. Also provided is a magnetogram to show the locations of the large sunspot, the surrounding plage, and the region of quiet Sun that is studied in all of the plots in the rest of the paper. The scatter plot of the two velocity maps shows that the magnetic field introduces an anomalous second component to the velocity field, reinforcing our reason to restrict all further analyses to the 


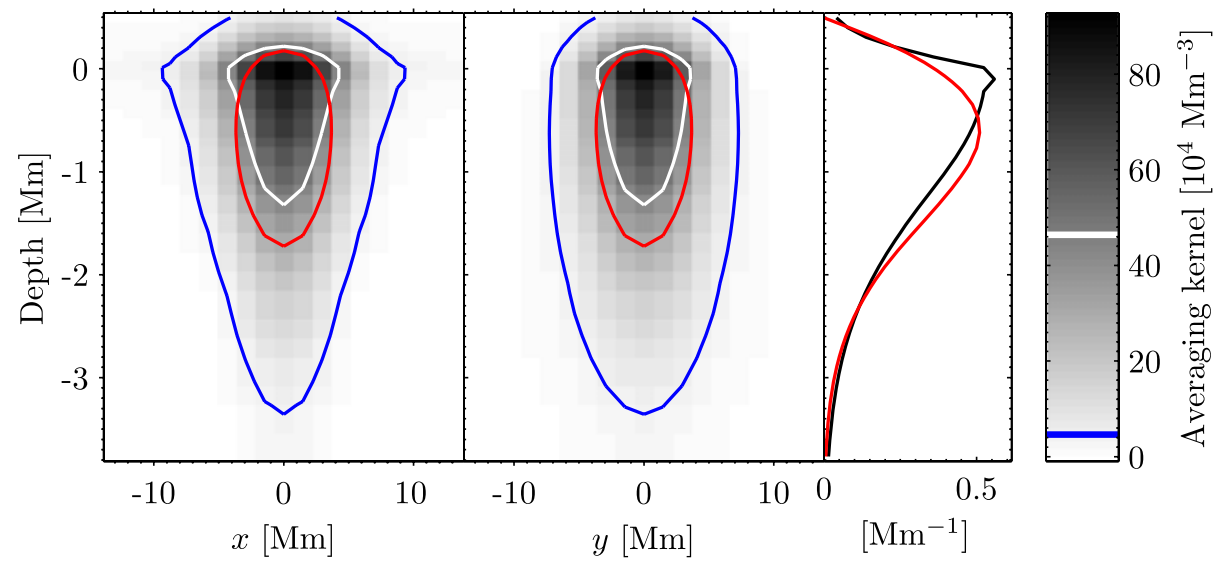

Figure 12 Vertical slice through a 3D averaging kernel $\left[{ }^{2+1 D} \mathcal{K}_{x}^{u_{x}}\right]$ after performing a $2+1 \mathrm{D}$ inversion for $u_{x}$ for a target depth of about $-1 \mathrm{Mm}$ below the solar surface. The left (middle) column is a slice along the $y=0(x=0)$ line. The red contours show the half-maximum value of the 3D target function, white contours show $50 \%$ of the maximum value of the averaging kernel, black contours show $-50 \%$ of the maximum value of the averaging kernel, and the blue (green) contours denote $\pm 5 \%$ of the maximum. In the rightmost panel, the red line is the 1D target function, and the black solid line is the $1 \mathrm{D}$ averaging kernel. The 1D weights $c^{n}$ and the noise estimate are given in Table 1.

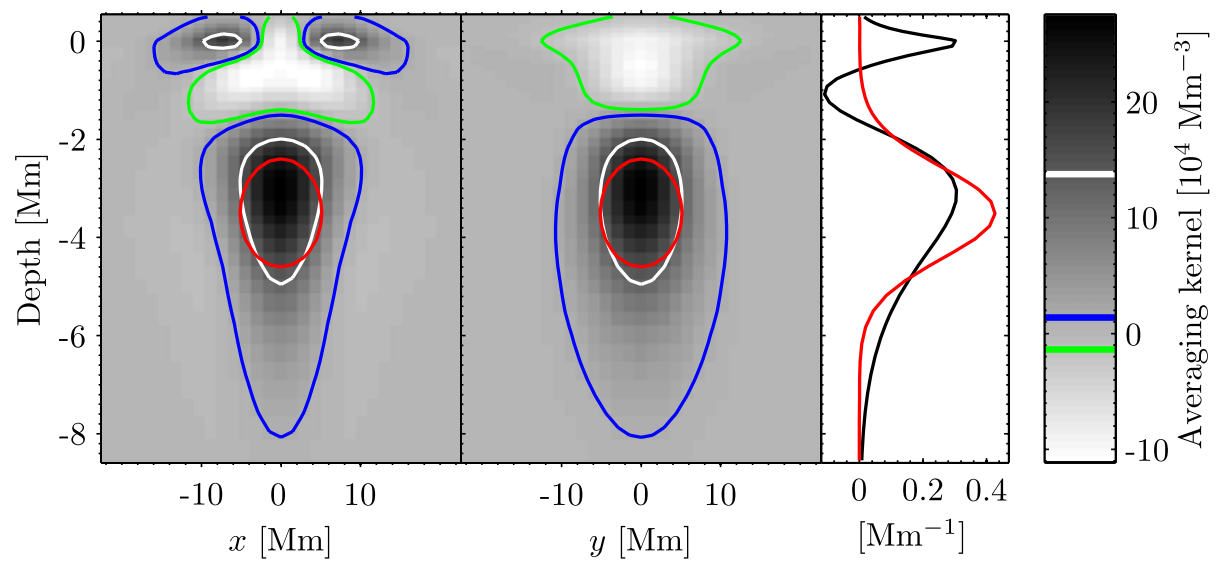

Figure 13 Vertical slices through an averaging kernel after performing a $2+1 \mathrm{D}$ inversion for $u_{x}$ for target depth $z_{t} \approx-3.5 \mathrm{Mm}$. The red contours show the half-maximum value of the $3 \mathrm{D}$ target function, white contours show $50 \%$ of the maximum value of the averaging kernel, black contours show $-50 \%$ of the maximum value of the averaging kernel, and the blue (green) contours denote $\pm 5 \%$ of the maximum. In the right panel, the red line is the $1 \mathrm{D}$ target function, and the black solid line is the $1 \mathrm{D}$ averaging kernel.

quiet Sun. That the inferred line-of-sight velocity is smaller than the Doppler velocity in not surprising. It is most likely attributable (Gizon, Duvall, and Larsen, 2000; Braun, Birch, and Lindsey, 2004) to the average depth over which the flows are measured. We cannot make a purely "surface" measurement with the available mode set.

A further example of the 2D inversion is shown in Figure 16. Here we show horizontalflow maps inferred from inverting individual-ridge travel times for $f, p_{1}$, and $p_{2}$ and for 24 hours. The color scale of these images is the horizontal divergence, obtained from a 


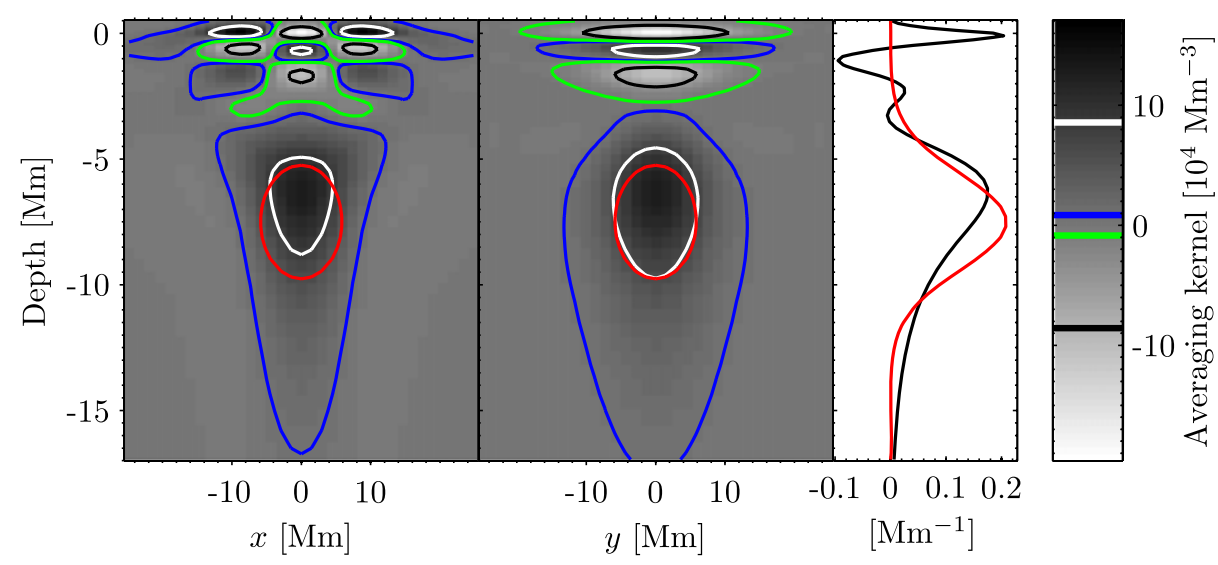

Figure 14 Vertical slices through an averaging kernel after performing a $2+1 \mathrm{D}$ inversion for $u_{x}$ for target depth $z_{t} \approx-7.5 \mathrm{Mm}$. The right panel shows the $1 \mathrm{D}$ averaging kernel and target function. The colors are the same as for Figure 13.

separate inversion as discussed in the following. The units of the horizontal divergence in this and all figures are inverse megaseconds $\left[\mathrm{Ms}^{-1}\right]$. In the $f$-mode map we see signatures of supergranulation with strong horizontal divergence in the centers of the supergranules and flow convergence at the cell boundaries. The outflow is generally in the $200-350 \mathrm{~m}$ $\mathrm{s}^{-1}$ range. The horizontal flows obtained from $p_{1}$ and $p_{2}$ travel times are weaker, as is the supergranulation signature. Maps for the $p_{3}$ and $p_{4}$ ridges have also been studied, but the noise begins to increase quickly with these modes at this resolution.

Obtaining the horizontal-flow divergence from a direct inversion such as shown by the color scale in the plots in Figure 16 is conveniently done in an OLA inversion such as this one. All that is needed is to use a different $2 \mathrm{D}$ target function such that the quantity that is inverted for is simply the horizontal divergence. The function we use (which would replace the function defined in Equation (15) in the 2D inversion) is

$$
\mathcal{T}^{\operatorname{div}_{\mathrm{h}}}(\mathbf{r})=\left(\frac{x}{2 \pi \sigma^{4}} \exp ^{-r^{2} / 2 \sigma^{2}}, \frac{y}{2 \pi \sigma^{4}} \exp ^{-r^{2} / 2 \sigma^{2}}, 0\right)
$$

where $\operatorname{div}_{\mathrm{h}}$ is a superscript label that denotes the horizontal divergence of the flow $\left[\nabla_{\mathrm{h}} \cdot \mathbf{v}_{\mathrm{h}}\right]$. Another useful quantity that we have studied is the vertical component of the flow vorticity $\left[\hat{z} \cdot \nabla_{\mathrm{h}} \times \mathbf{v}_{\mathrm{h}}\right]$. The associated target function, labeled with the superscript vort ${ }_{z}$, can be shown to be

$$
\mathcal{T}^{\text {vort }_{z}}(\mathbf{r})=\left(\frac{-y}{2 \pi \sigma^{4}} \exp ^{-r^{2} / 2 \sigma^{2}}, \frac{x}{2 \pi \sigma^{4}} \exp ^{-r^{2} / 2 \sigma^{2}}, 0\right) .
$$

An example map of the vertical vorticity obtained directly from a 2D inversion is shown in Figure 17. We have studied plots of the divergence and vorticity at different resolutions and checked that the inverted quantities using these two types of targets and the direct numerical computation of these quantities using inferred horizontal vector flows agree reasonably well. The advantage of computing them directly from an inversion is that we are able to determine the noise and spatial resolution properly. 

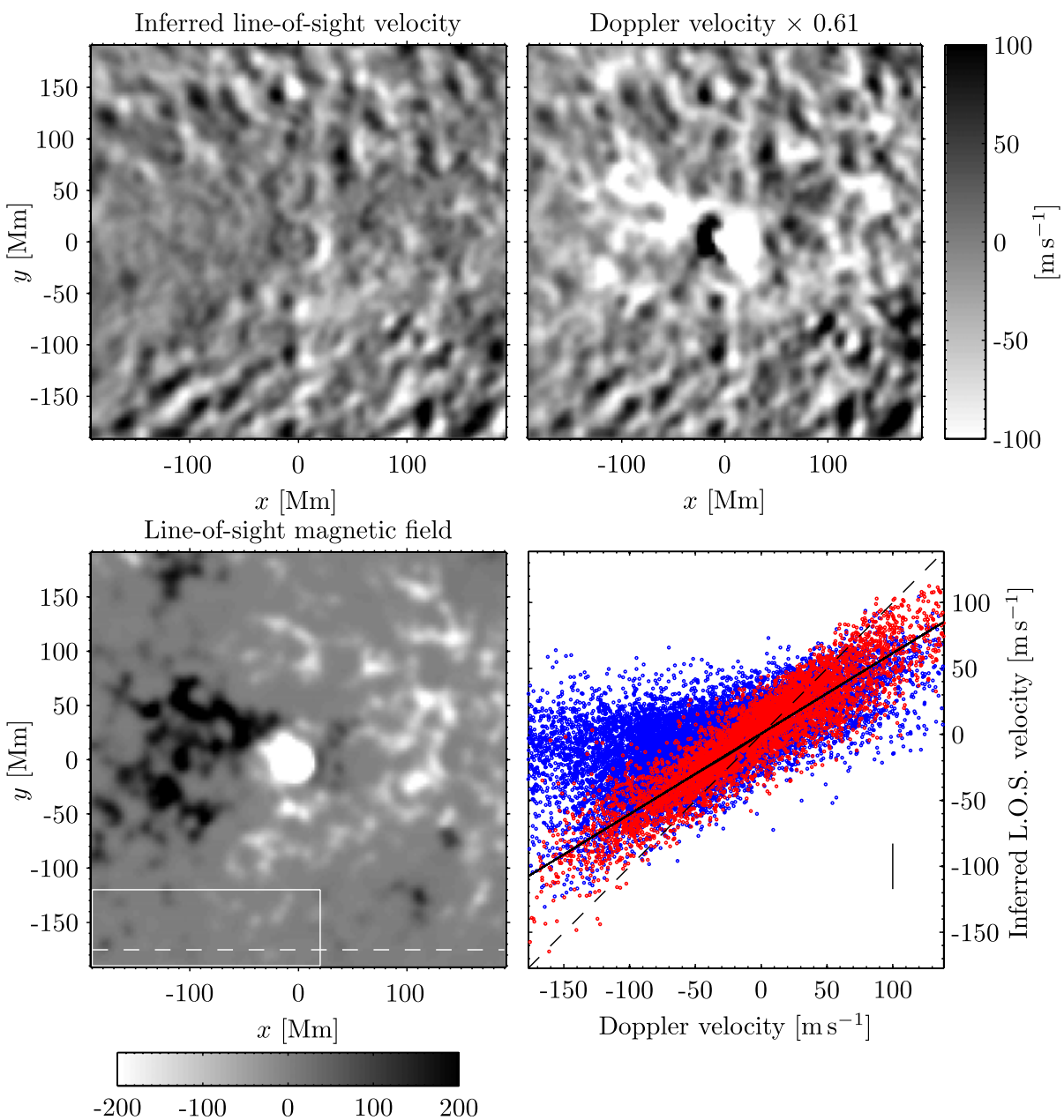

Figure 15 Test of the 2D inversion. The top panels show a comparison of the inverted flows with the direct Doppler data for a map centered at $30^{\circ}$ west of disk center. The top-left panel is the inferred vector flow map after projection onto the line-of-sight vector at each pixel for an inversion using $f$ modes and 24 hours of data. The resolution is about $7 \mathrm{Mm}$. The Doppler map is an average over one day of MDI full-disk data, smoothed with the 2D averaging kernel from the inversion and multiplied by the slope 0.61 of the best-fit line through the scatter plot (panel below) to allow for direct comparison with the inverted map. The lower left panel is the one-day-averaged (truncated) MDI magnetogram with a sunspot visible in the middle. The units are in gauss. The white box outlines the quiet-Sun region analyzed for all of the plots in the rest of the paper. The white dashed line shows the location of the slice for the flows in Figure 24. The lower right panel shows a scatter plot of the velocity maps, where red (blue) dots represent pixels of less (more) than $|10|$ G. The correlation is 0.9 for the nonmagnetic data. The dashed line is $y=x$, and the solid line is a fit to the nonmagnetic data (with a slope of 0.61$)$. The small vertical line represents the $\pm 1 \sigma\left(\sigma=17 \mathrm{~m} \mathrm{~s}^{-1}\right)$ noise in the flow estimation.

\subsection{Tests of the $2+1 \mathrm{D}$ Inversion}

A consistency test of the full $2+1 \mathrm{D}$ inversion is shown in Figure 18 . We study flows in a quiet-Sun region obtained from two independent inversions using 24 hours of data. Shown are the horizontal flows computed from inversions for $v_{x}$ and $v_{y}$ along with the line-of-sight 

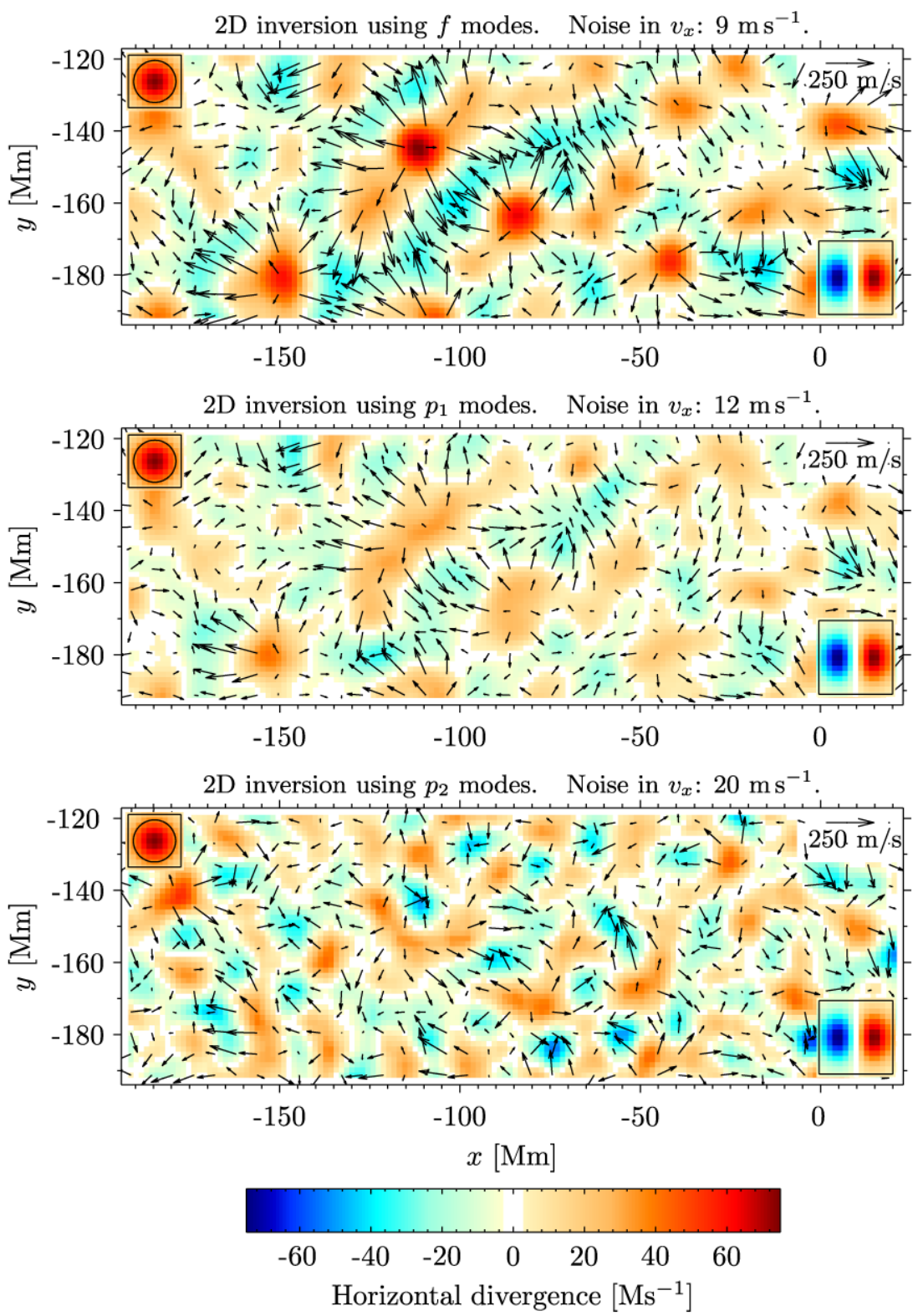

Figure 16 Horizontal flows and divergence from a 2D inversion using 24 hours of data for the $f, p_{1}$, and $p_{2}$ ridges. In each panel, the arrows denote the horizontal flows (obtained from inverting for $v_{x}$ and $v_{y}$ ) for 24 hours of data, and the color scale is the horizontal flow divergence in units of inverse megaseconds, obtained from a separate inversion as described in the text at the end of Section 8.1. The $x$-component of the $2 \mathrm{D}$ averaging kernel from the flow inversion is plotted in the box in the upper left of each panel and the FWHM, outlined by the circle, is $11.6 \mathrm{Mm}$. The $x$-component of the 2D averaging kernel for the horizontal divergence is given by the quantity in the lower right box (see Equation (34)). Note the strong supergranular flows in the $f$-mode map, which gradually weaken as the modes probe deeper layers of the convection zone. The correlation of the $f$-mode map with the $p_{1}$-mode map is 0.88 , and that between the $f$-mode and $p_{2}$-mode maps is 0.35 . The noise in $v_{x}$ is given for each panel. The noise in the horizontal divergence inversion is 10 , 12 , and $13 \mathrm{Ms}^{-1}$ for the top, middle, and bottom panels, respectively. 


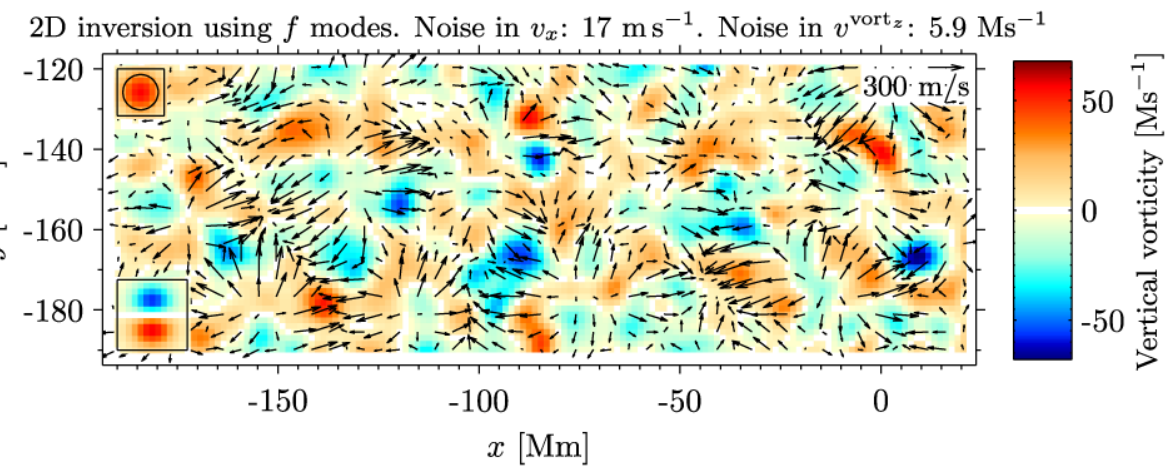

Figure 17 2D inversions for the vertical vorticity and horizontal flows in the quiet Sun. The arrows denote the horizontal flows and the color scale is the vertical vorticity obtained from a separate inversion using 24 hours of data. The $x$-component of the averaging kernel from the inversion for the horizontal flows is given in the upper left box, and the $x$-component of the averaging kernel for the vorticity inversion, which matches the target function from Equation (35), is shown in the lower left box.

magnetic field (color scale). In the inversion corresponding to panel (a), we only invert $p_{1}$ travel times. We then attempt to target the $p_{1}$ averaging kernel by using all of the other available mode-ridge kernels except $p_{1}$, in other words, $f, p_{2}, p_{3}$, and $p_{4}$. Once a similar averaging kernel is found, we invert the corresponding $f, p_{2}, p_{3}$, and $p_{4}$ travel times, and the resulting flows are shown in Figure 18(b). The 1D averaging kernels for each case are given in the panel on the bottom right. The maps are quite similar (correlation $=0.82$ ), and the differences could be due simply to the differences in the averaging kernels. In these maps the supergranule-scale flows are evident, and the magnetic field is concentrated at the boundaries of the supergranules as expected. A best fit through a scatter plot of the data that takes into account the noise in both variables gives a slope of 0.83 . The magnetic field does not introduce any anomalous component in the scatter as it did for the full-map study in Figure 15, confirming that this is a quiet-Sun region for our purposes. We have also used this test to see whether we can recover an $f$-mode map by inverting the four available acousticmode travel-time sets. For as closely as we are able to match the averaging kernels, it is successful. The same conclusion can be drawn for the other possible cases when noise is not a limiting factor.

In all of the plots studied so far, we have shown flows obtained from one day of travel times. In Figure 19, we compare horizontal flows at a depth of $1 \mathrm{Mm}$ below the surface from inversions for different observation durations. The panels show inversions for 6 to 24 hours of data in six-hour intervals. Also shown is the horizontal divergence computed numerically (since we have not yet computed inversions directly for horizontal divergence at depth). What is evident is that even with six hours of data and a resolution of about $9 \mathrm{Mm}$, the noise level is reasonable and features are seen that have much in common with the one-day map. The correlation between the 6-hour and 24-hour maps is still about 0.8. It is encouraging that the supergranulation signal at this depth is not dominated by noise for six hours of data.

We now compare horizontal flows at three different depths from the full $2+1 \mathrm{D}$ inversion. Figure 20 shows the flow field at depths of $1 \mathrm{Mm}$ (top), $2.6 \mathrm{Mm}$ (middle), and 3.7 Mm (bottom) below the surface using 24 hours of data. The color scale is the horizontal divergence computed by numerical differentiation of $v_{x}$ and $v_{y}$. The 1D inversion weights for each map are given in Table 1. The flows at the different depths in Figure 20 are not too unlike the individual ridge flows shown in Figure 16, and inspection of the 1D inversion 
(a) 2D inversion using $p_{1}$ modes. Noise in $v_{x}: 19 \mathrm{~m} \mathrm{~s}^{-1}$

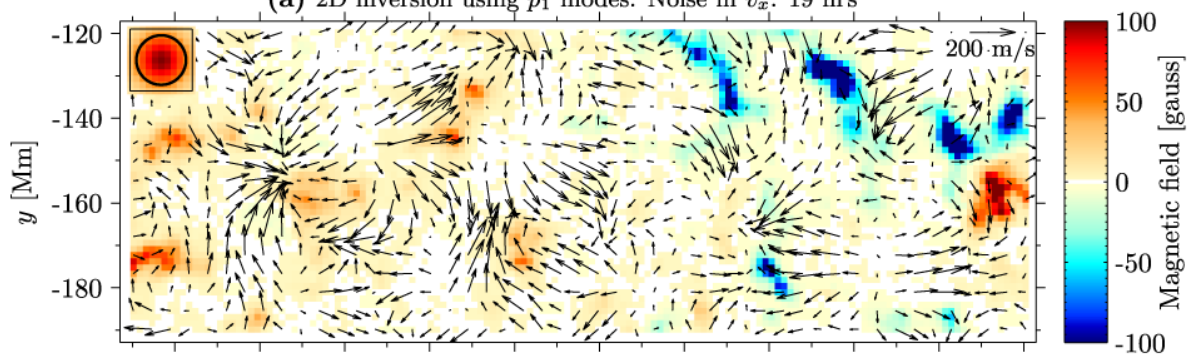

(b) $2+1 \mathrm{D}$ inversion using $f, p_{2}, p_{3}$, and $p_{4}$ modes. Noise in $v_{x}: 17 \mathrm{~m} \mathrm{~s}^{-1}$
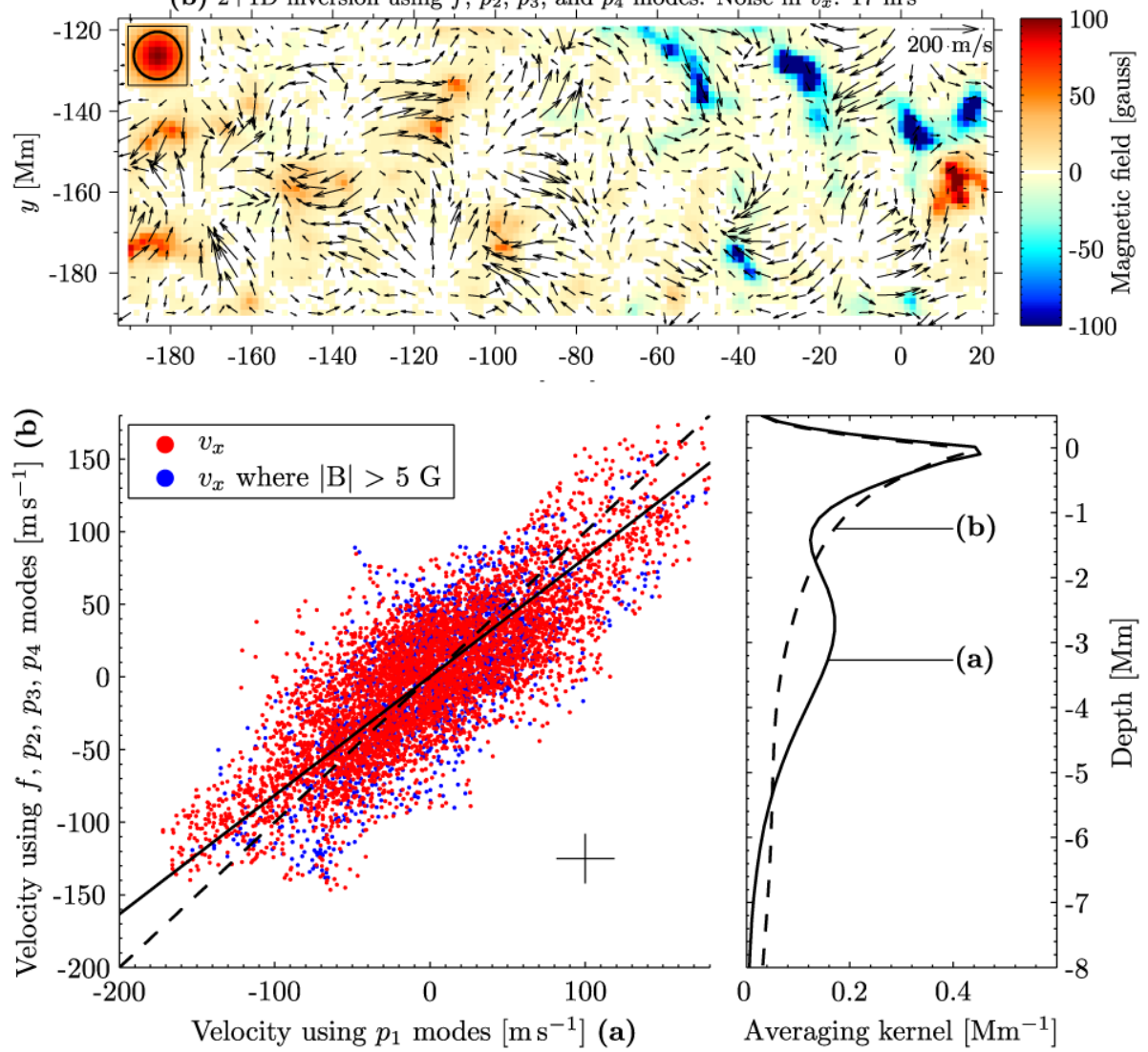

Figure 18 Test of the full $2+1 \mathrm{D}$ inversion for (a) the flows from a $2 \mathrm{D}$ inversion using only the $p_{1}$-mode ridge travel times and (b) the flows from a $2+1 \mathrm{D}$ inversion using travel times from all other mode ridges (no $p_{1}$ modes) by attempting to target the same averaging kernel. The arrows denote the horizontal flows (obtained from inverting for $v_{x}$ and $v_{y}$ ) for 24 hours of data, and the color scale is the truncated magnetic field from MDI. The $x$-component of the $2 \mathrm{D}$ averaging kernel is plotted in the box in the upper left, and the FWHM $=11 \mathrm{Mm}$ is outlined by the circle. The scatter plot shows the flows from panel (a) vs. (b), where the red (blue) dots are the values in the maps for which the magnetic field of the pixel is less (greater) than $|5| \mathrm{G}$. The dashed line is the $y=x$ line and the solid line is the best fit through the nonmagnetic data (slope $=0.83$ ), taking account of the errors on each axis. The $1 \sigma$ error bars for each measurement are denoted by the cross in the lower right of the plot. The correlation in the scatter is 0.82 . The bottom right panel shows the $1 \mathrm{D}$ averaging kernel (solid line) for panel (a) and the 1D averaging kernel (dashed line) for panel (b). The $1 \mathrm{D}$ inversion weights for these two inversions are listed in Table 1. 

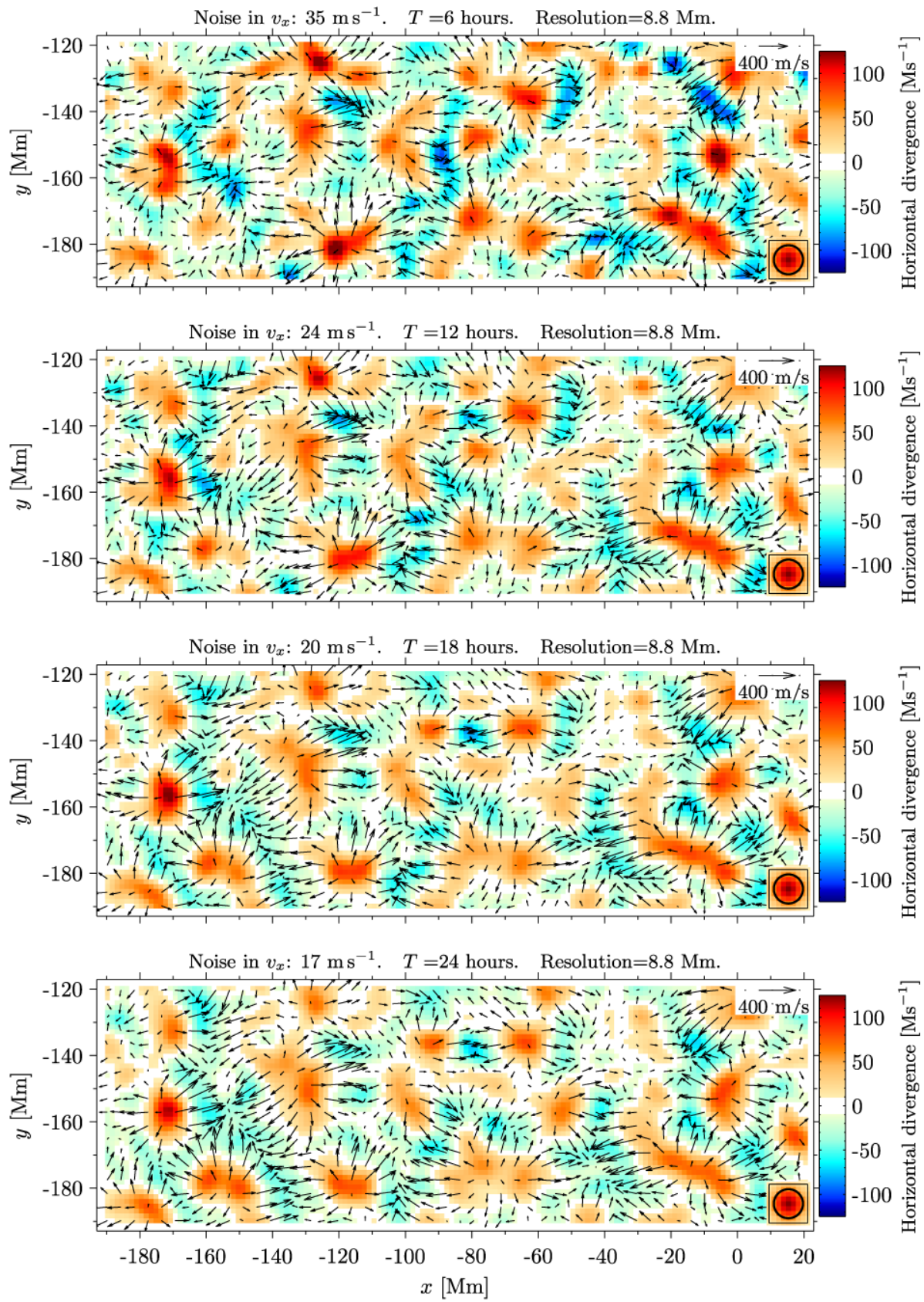

Figure 19 Comparison of flows for different observation durations $[T]$ obtained from $2+1 \mathrm{D}$ inversions at a depth of $1 \mathrm{Mm}$ beneath the surface. The arrows are the inferred horizontal flows and the color scale is the horizontal divergence computed numerically from $v_{x}$ and $v_{y}$. The total observation time increases from top to bottom in six-hour intervals, but the horizontal resolution remains fixed at about $9 \mathrm{Mm}$. The correlation of the 6-hour map with the 24-hour map is 0.78 , whereas the correlation of the 12-hour (18-hour) map with the one-day map is $0.9(0.97)$. Note that the divergence scale is the same for each panel. 

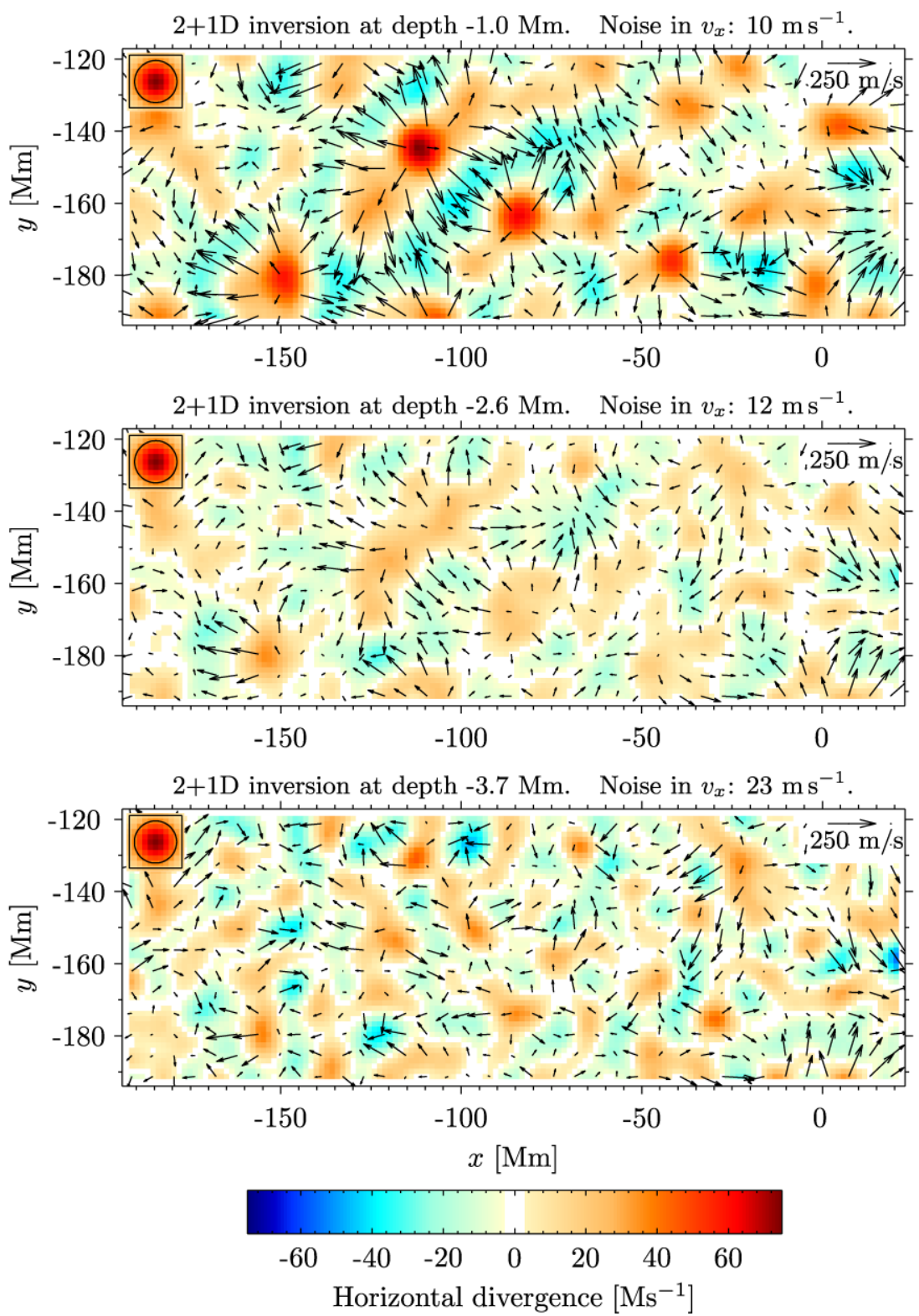

Figure 20 Comparison of horizontal flows (arrows) at different depths from a $2+1 \mathrm{D}$ inversion for one day of data. The background color scale is the horizontal divergence to emphasize the various flow structures and is obtained by numerical differentiation of the 2D flows. The $x$-component of the $2 \mathrm{D}$ averaging kernels are shown in the upper left and all have FWHM $=11.6 \mathrm{Mm}$. The correlation of the $x$-component of the flows at depths of -1 and $-2.6 \mathrm{Mm}$ is 0.77 . The correlation of the $x$-component of the flows at depths of -1 and $-3.7 \mathrm{Mm}$ is 0.33 . The $1 \mathrm{D}$ inversion coefficients for each depth inversion are provided in Table 1 . 


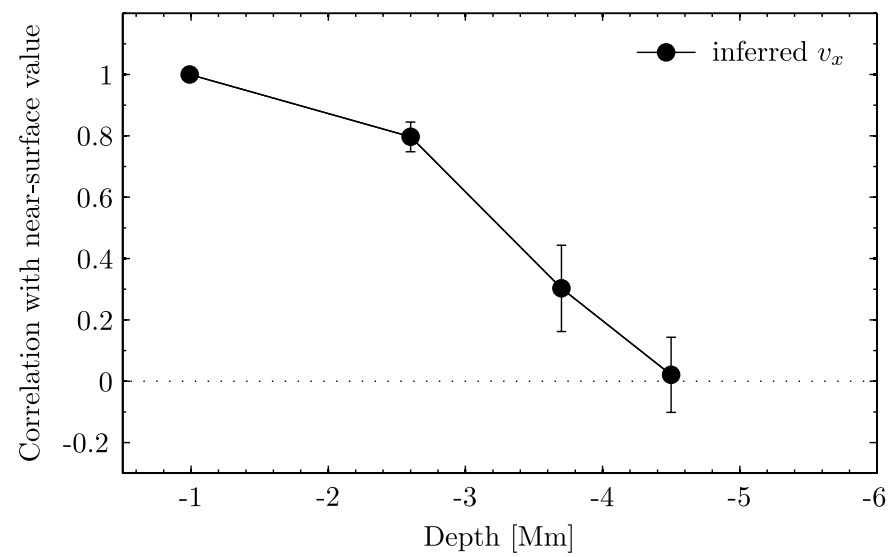

Figure 21 Correlation coefficient with respect to the near-surface value of the inferred $v_{x}$ flows at different depths using 24 hours of data. The values represent the average over five days of the flows measured in the area of the quiet Sun used throughout this paper. The $1 \sigma$ error bars are plotted at each depth point, obtained from the standard deviation in the correlation coefficients over the five days. The "near-surface value" for this case is at a depth of $-1 \mathrm{Mm}$.

weights confirms that this should be the case. This figure also demonstrates that combining the maps with the 1D inversion not only gives a good estimate of the target depth but also generally lowers the noise levels.

We have studied the correlation of maps of $v_{x}$ and $v_{y}$ such as those in Figure 20 at different depths with the near-surface map and averaged over five days. The results are provided in Figure 21. Each measurement is for 24 hours of data, and the error bars are obtained by studying the variance in the correlation values. The correlation steadily decreases as we go deeper, and it seems to disappear at about $5 \mathrm{Mm}$ below the surface. However, the noise levels at these depths are quite large and we can draw no other specific conclusions at this time. This is consistent with recent studies on realistic numerical simulations using timedistance helioseismology (Zhao et al., 2007) and helioseismic holography (Braun et al., 2007). In fact, Braun et al. (2007) note that “... supergranule-sized flows are essentially undetectable using current methods below depths around $5 \mathrm{Mm}$..." using 24 hours of data or less. We confirm this conclusion here and note that similar results have also been found with direct modeling techniques (Woodard, 2007).

We have also studied the day-to-day correlation of the $v_{x}$ and $v_{y}$ maps at various depths. If we were predominantly measuring noise, there would be no significant correlation from one 24-hour period to the next. Computing an average day-to-day correlation over seven days of data for the $-1 \mathrm{Mm}$ depth maps gives a value of about 0.4. For the $-3.7 \mathrm{Mm}$ depth, we find a 0.26 correlation, and at a depth of $-6 \mathrm{Mm}$, about a 0.1 correlation. This again demonstrates that there is plenty of near-surface flow signal when 24-hour averages are studied, presumably owing to supergranulation (Gizon and Birch, 2004), which then quickly decreases with depth.

\subsection{Vertical Flows}

It has proven difficult in helioseismology to accurately measure the vertical component of the velocity near the surface owing in part to its small magnitude compared to the other components. In fact, in many helioseismic inversions for flows, $v_{z}$ is obtained approximately by 
2D inversion using $f$ modes. Noise in $v_{x}: 6.4 \mathrm{~m} \mathrm{~s}^{-1}$. Noise in $v_{z}: 8.3 \mathrm{~m} \mathrm{~s}^{-1}$.

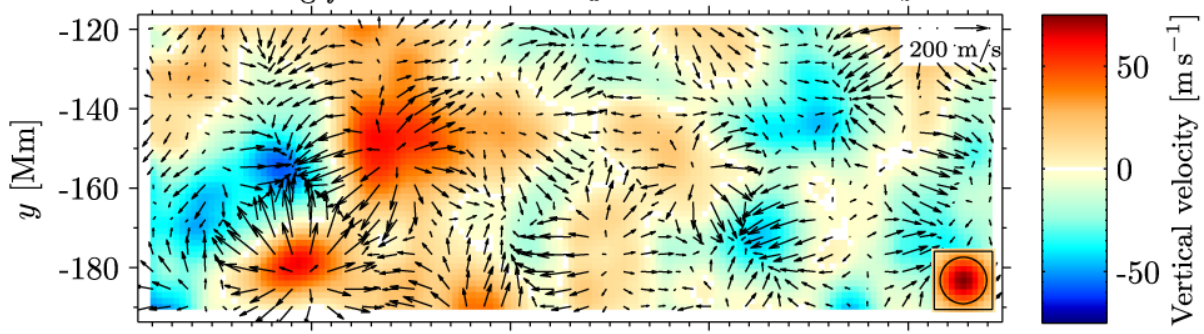

2D inversion using $p_{1}$ modes. Noise in $v_{x}: 8.5 \mathrm{~m} \mathrm{~s}^{-1}$. Noise in $v_{z}: 11 \mathrm{~m} \mathrm{~s}^{-1}$.

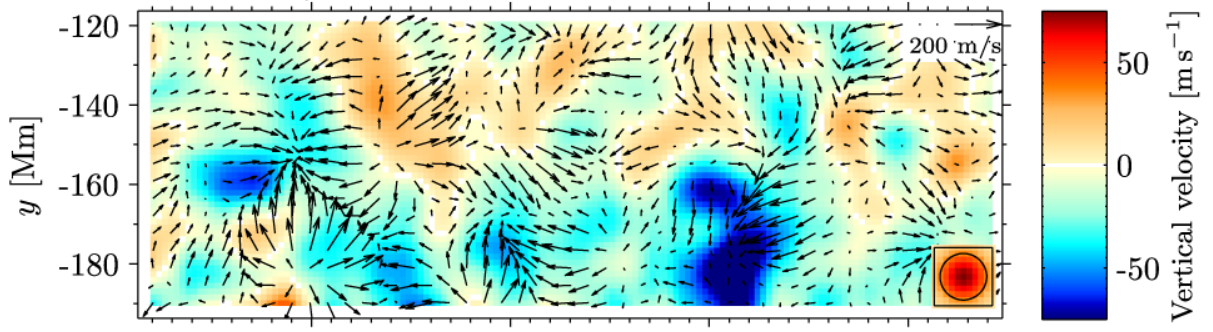

$2 \mathrm{D}$ inversion using $p_{2}$ modes. Noise in $v_{x}: 14 \mathrm{~m} \mathrm{~s}^{-1}$. Noise in $v_{z}: 8.9 \mathrm{~m} \mathrm{~s}^{-1}$.

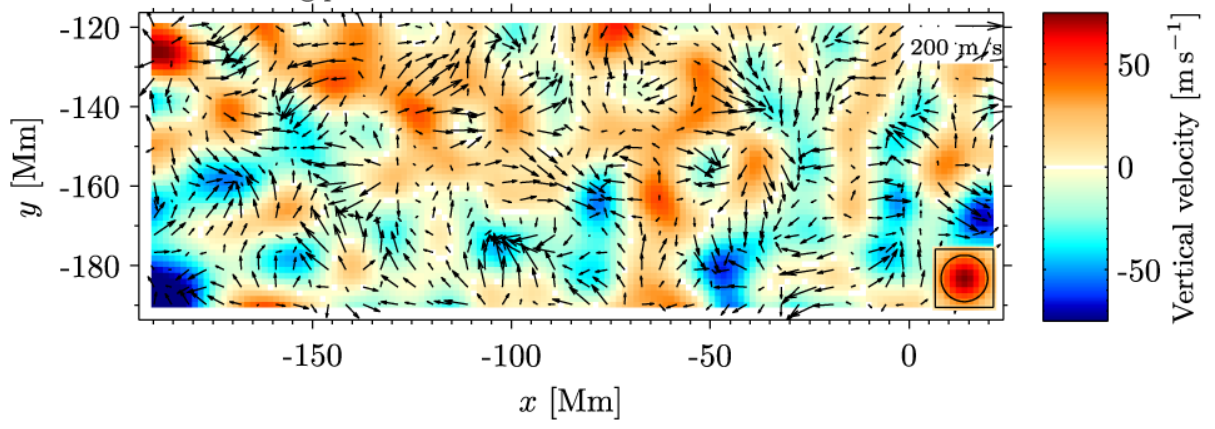

Figure 22 Vertical flows (color scale) and horizontal flows (arrows) in the quiet Sun for a 2D inversion using $f$ modes (top), $p_{1}$ modes (middle), and $p_{2}$ modes (bottom). These flows were obtained by using 48 hours of data. A positive vertical velocity means an upflow. The $z$-component of the $2 \mathrm{D}$ averaging kernel from the inversion for $u_{z}$ is given by the quantity in the box in the lower right. The noise for all of the measurements is indicated. The correlations of these particular $p_{1}$ and $p_{2}$ vertical flows with the $f$-mode map are about 0.3 and 0.2 , respectively. The region of the Sun used here is outlined by the white box in Figure 15. The color scale is the same in each panel to ease comparison.

computing the horizontal-flow component and then invoking mass conservation from the continuity equation (see Komm et al., 2004, for an example in ring-diagram analysis). Another source of difficulty in these measurements has been associated with cross-talk effects, whereby the inversion (or sensitivity kernel) becomes insensitive to differences between upflows and convergence and between downflows and divergence (Zhao et al., 2007). These inversions, usually based on the ray approximation, have no obvious means of constraining the cross-talk. Since we have available Born sensitivity kernels for $v_{z}$ and an inversion procedure that measures each flow component while minimizing the cross-talk with the others, we can obtain vertical flows directly and with the assurance that they are relatively indepen- 


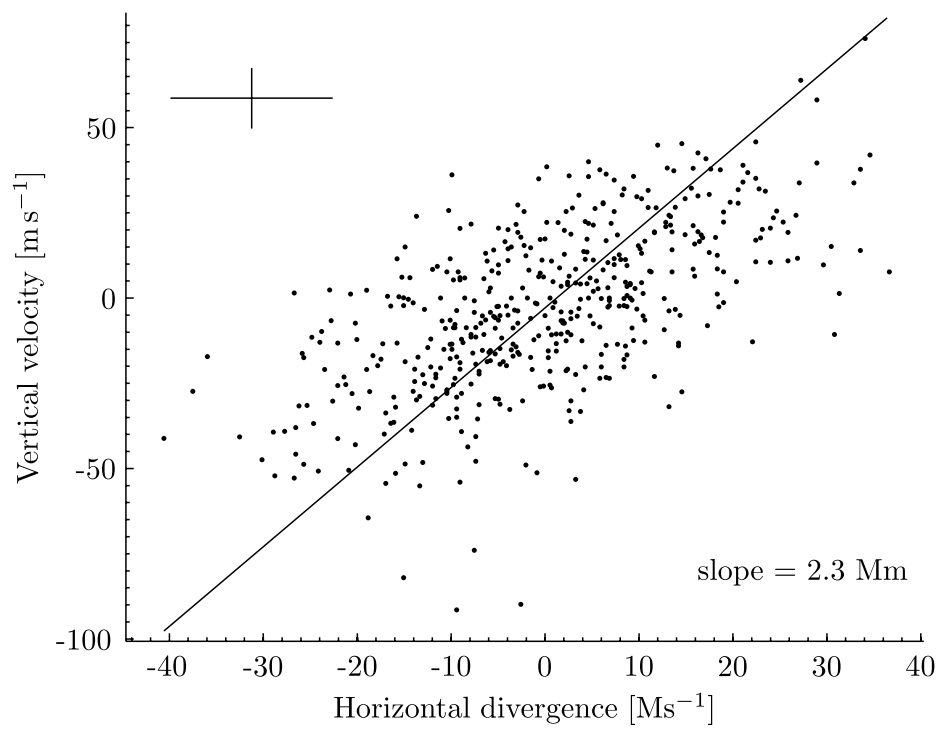

Figure 23 Vertical velocity versus horizontal divergence for flows using $p_{2}$ modes measured over two days for the same region of the Sun as shown in Figure 22. The vertical flows and the horizontal divergence were computed from two separate inversions. The correlation coefficient is 0.62 . The line shows a best fit through the scatter by taking into account the noise on each axis, which is $9 \mathrm{~m} \mathrm{~s}^{-1}$ for $v_{z}$ and $9 \mathrm{Ms}^{-1}$ for the divergence, indicated by the cross in the upper left of the figure. The slope of the best-fit line is a rough proxy for the density scale height at the implied depth.

dent of the horizontal measurements. This is clearly demonstrated in the averaging kernels of Figures 6 and 7. We note that we have so far only tested the 2D inversion for $v_{z}$; thus, the maps shown here are for individual ridge measurements.

There tends to be much more relative noise in the measurements of vertical velocity, and therefore in Figure 22 we show the vertical component of the velocity as the color scale averaged over two days from a $2 \mathrm{D}$ inversion (with the noise going as $T^{-1 / 2}$, where $T$ is the observation time). The top panel of Figure 22 is for the $f$-mode ridge, the middle panel for $p_{1}$, and the bottom panel for $p_{2}$. Also shown are the corresponding horizontal flows given by the arrows. One generally sees a good correspondence in all maps between the vertical upflows and horizontal outflows, as well as between downflows and horizontal inflows. Analysis of many similar maps shows that the speeds of the vertical flows in the center of supergranules near the surface are on average about $15-20 \%$ of the speeds of the horizontal outflow in the supergranules, slightly higher than recent observations might suggest (Hathaway et al., 2002).

To understand whether the inferred vertical flows at these depths for $p_{2}$ are reasonable, we compare them with maps of the horizontal divergence, obtained from a separate and independent inversion of this quantity as explained in Section 8.1. The vertical component of the flow and horizontal divergence are proportional if one writes down an approximate continuity equation whereby one neglects the horizontal variations in the density and the vertical gradient of the vertical flow. The scaling factor is the density scale height. In Figure 23 we show a scatter plot of $v_{z}^{p_{2}}$ against the horizontal divergence inferred from inverting $p_{2}$ travel times for the same region of the Sun as in Figure 22. The correlation coefficient is 0.62. The noise in $v_{z}$ is $9 \mathrm{~m} \mathrm{~s}^{-1}$ and the noise is $9 \mathrm{Ms}^{-1}$ for the divergence measurement. The slope 
of the best-fit line, obtained by using the noise information in both variables, gives a value of about $2.3 \mathrm{Mm}$. This value is in the range of the density scale height for the implied depth range of these vertical flows. We have also studied the correlation of vertical flows maps with horizontal divergence maps for the $f$-mode and $p_{1}$-mode cases. The values are always in the range of $0.6-0.7$.

Another interesting question is how well the near-surface vertical flows are correlated with deeper vertical flows. Since we have so far only implemented the 2D inversion scheme for vertical flows, we take different mode ridges as a proxy for depth. We correlate the 24-hour $f$-mode $v_{z}$ map with the $p_{1}$ and $p_{2}$ maps, averaged over seven days, and find correlations of about 0.3 and 0.2 , respectively. In addition, as was described previously for the horizontal component, we have also studied the day-to-day correlations of the vertical flows averaged over seven days of data. The average day-to-day $f$-mode map correlation is 0.15 , and it is 0.2 for $v_{z}^{p_{1}}$ and 0.15 for $v_{z}^{p_{2}}$. This demonstrates again that the $v_{z}^{n}$ inversions are measuring long-lived flow structures and not just noise.

Finally, in Figure 24 we show the culmination of our main results. It is a slice in depth through the horizontal divergence with overplotted $v_{z}$ information. The inversion to obtain these flows used 72 hours of travel times. The slice is along a line through the quiet Sun (shown by the dashed white line in the magnetogram of Figure 15) chosen because of the presence of many near-surface large-scale flow structures. Inversions for $v_{x}$ at different target depths were performed, the numerical divergence $\partial_{x} v_{x}$ was computed, and the results are given by the color scale. The color scale is such that a positive divergence means an outflow. The $v_{z}$ flows were obtained by using $f, p_{1}$, and $p_{2}$ travel times, and the magnitudes and directions are shown by the arrows. Since we have not yet implemented a 1D depth inversion for $v_{z}$, we roughly determine the three depth locations by computing the average depth over which the dominant modes of these three ridges probe. We emphasize that these placements are only approximate. We also plot the $1 \sigma$ noise levels of $v_{z}$ at each depth. We see that over the whole depth range, the horizontal inflows (outflows) generally correspond to vertical downflows (upflows). What one would expect is to see relatively stronger vertical flows where the divergence is strongest in absolute value. This is for the most part the case. We emphasize that these good correlations are likely not due to cross-talk contamination, which tends to diminish as one moves further below the surface (see Section 5.2). Note also the presence of large-scale structures that live for at least three days.

\section{Summary and Conclusions}

We have presented in detail a fully consistent procedure for inverting helioseismic travel times to infer vector flows in the upper convection zone of the quiet Sun. Travel-time sets are measured for all modes that have the same radial order, in other words, along the ridges in the power spectrum. The travel times are constructed by using an analog to the common point-to-annulus geometry for 20 annulus radii (up to about $30 \mathrm{Mm}$ ). Three-dimensional Born sensitivity kernels for the same travel-time definition and ridge filtering are computed. In addition, the noise-covariance properties of the travel times are calculated. Based on the separability of the sensitivity kernels into horizontal and vertical components owing to the ridge filtering, the inversion is formulated in two steps: The first step solves the $2 \mathrm{D}$ horizontal problem and the second step solves the 1D depth inversion. Optimal sets of weights are chosen from both inversions, such that the final averaging kernel is regularized in the horizontal and vertical directions. We have provided examples of averaging kernels, which are useful for understanding where the sensitivity of the inversion is located, as well as for 

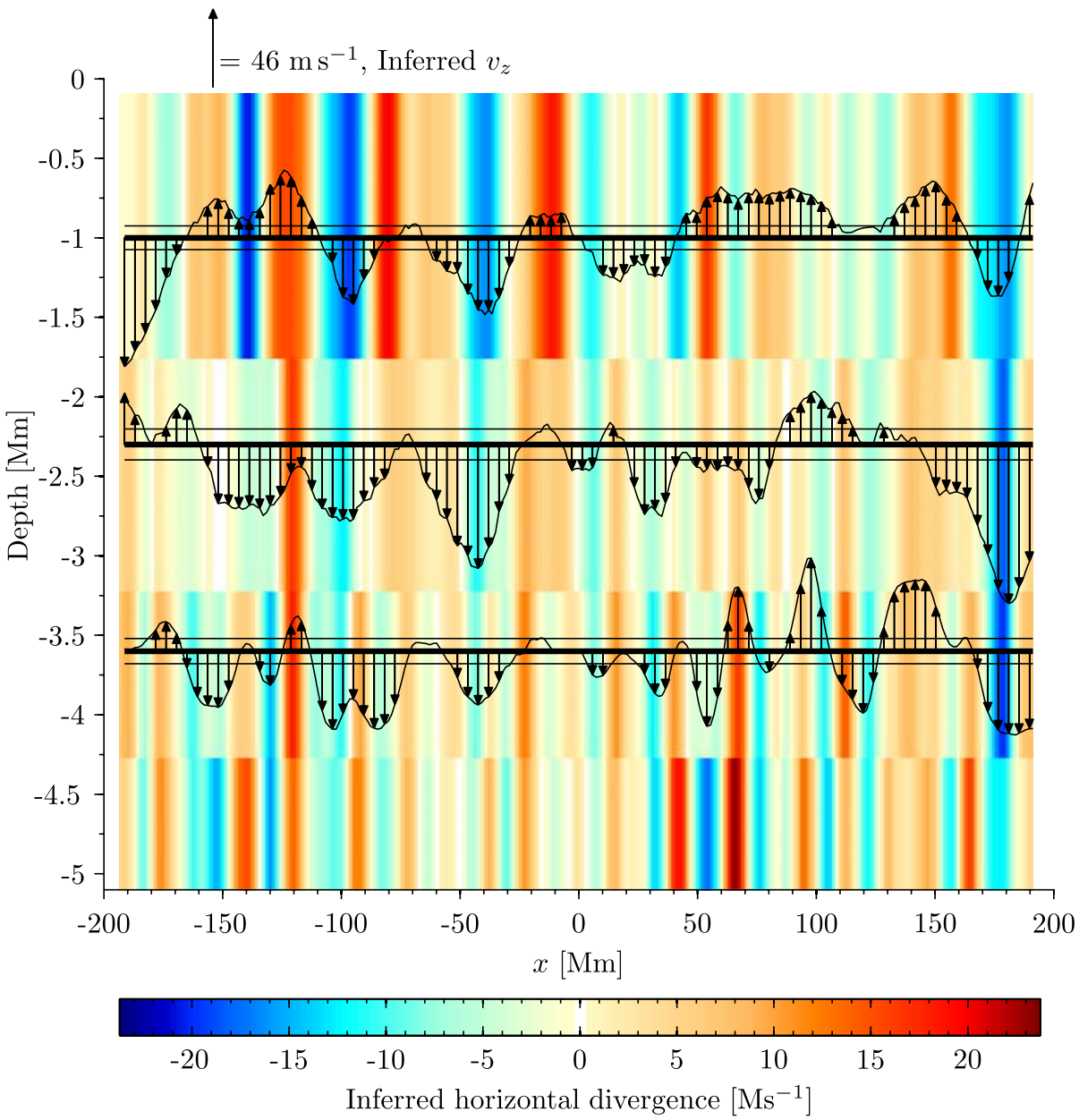

Figure 24 Depth slice through a flow map from inversions using three days of data. The horizontal divergence of $v_{x}$ is shown by the color scale and the arrows represent the velocity of the vertical flows at three different depths. The three thick black horizontal lines denote the approximate depth locations of the $v_{z}$ measurements, which are found by computing the center of mass of the $1 \mathrm{D}$ sensitivity functions for the first three ridges (see Figure 9). The top curve shows $v_{z}^{f}$, the middle $v_{z}^{p_{1}}$, and the bottom $v_{z}^{p_{2}}$. The divergence map was obtained by computing maps of $v_{x}$ at several different depths where each depth block, centered about the target depth, has a horizontal spatial resolution of $<10 \mathrm{Mm}$. Then the numerical divergence was calculated. A positive divergence means an outflow. The $\pm 1 \sigma$ noise for the three $v_{z}$ measurements are given by the two thin lines above and below the thicker depth indication line, and all have values less than $10 \mathrm{~m} \mathrm{~s}^{-1}$. Note at the top the reference scale arrow for $v_{z}$.

determining the amount of cross-talk among all of the flow components. It was found that the cross-talk is reasonably small because the inversion procedure attempts to minimize its effect by the use of certain constraints. We furthermore obtain consistent estimates of the noise and spatial resolution on the measured velocities. For practical reasons, the inversion technique is convenient because directly inverting for other quantities such as the horizontal divergence of the flows or the vertical vorticity only requires a change of the target function. 
High-resolution example flow maps have been studied and tested. We have restricted ourselves to quiet-Sun cases. These maps all have horizontal spatial resolution of less than about $11 \mathrm{Mm}$, or about the $p_{1}$-mode wavelength at $3 \mathrm{mHz}$. The recovered flow speeds are below the limits for which a linearized theory of travel times is valid (Jackiewicz et al., 2007a). We have tested the inversion in several straightforward ways. We have shown that using independent measurements and similar averaging kernels gives consistent results. We have also been able to obtain high correlations $(\approx 0.9)$ with the Doppler-velocity data after projecting the inferred horizontal flows onto the line-of-sight vector and ignoring pixels with strong magnetic fields.

We also found that the correlation coefficient of 24 hours of inferred horizontal flows from day to day on average is about 0.4 near the surface and about 0.1 down to about $6 \mathrm{Mm}$ below the surface. This is consistent with the conclusion that we are not just measuring noise. However, we find that the correlation of flows at a particular depth with the surface flows falls off quite rapidly and disappears near $\approx 5 \mathrm{Mm}$ beneath the surface, where we do not see any more evidence of supergranulation. Similar results have also recently been found with numerical simulations using time - distance helioseismology (Zhao et al., 2007) and holography (Braun et al., 2007). It could be that for 24 hours and at these depths the supergranulation signal is completely masked by noise (Braun et al., 2007; Woodard, 2007).

We have shown a direct inversion for the vertical component of the velocity using acoustic and surface-gravity waves. The results are in agreement with the overall behavior of the horizontal flows, and since the cross-talk among $v_{x}, v_{y}$, and $v_{z}$ has been made small, we are fairly confident that the vertical flows are real. The vertical flows have also been compared to independent inversions for the horizontal divergence, and the values are in the expected ranges. We find that the upflow speeds in the center of supergranules are approximately $15-20 \%$ of the horizontal outflow speeds. Studying the day-to-day correlations of vertical flow maps also leads us to believe that the signal is above the noise.

Another way to validate many of the findings that we have reported would be to invert the available artificial velocity data from realistic numerical simulations of solar convection (Benson, Stein, and Nordlund, 2006). Even though for this inversion the averaging kernels give a complete picture of how the data are spatially averaged and therefore a good idea of what the final answer will look like - a nice feature of OLA-type inversions - we intend to carry this out in the near future to study the role that noise plays in the interpretation of the results.

Of course, we are undertaking many improvements to the inversion presented here. One obvious deficiency is the small set of modes that we have used. Such a limited number does not allow us to obtain many independent target depths, nor any substantially deep ones. More ridges, combined with utilizing the spatial-frequency content of the waves in each ridge in a more sophisticated way, would help us to obtain better, and deeper, averaging kernels.

There are several other improvements currently being studied, including ways to minimize the cross-talk among flow components as much as possible by constructing different types of constraints in the inversion procedure. Also, kernels that take into account the lineof-sight projection are almost certain to be necessary for inverting data well away from disk center. We already have some of these kernels available (Jackiewicz, Gizon, and Birch, 2006).

Acknowledgements We thank T. Duvall Jr. for helpful discussions and for providing the data set used in the analysis. We also gratefully acknowledge critical comments from a referee that significantly improved this paper. SOHO is a collaboration between NASA and ESA. 
Open Access This article is distributed under the terms of the Creative Commons Attribution Noncommercial License which permits any noncommercial use, distribution, and reproduction in any medium, provided the original author(s) and source are credited.

\section{References}

Basu, S., Antia, H.M., Tripathy, S.C.: 1999, Ring diagram analysis of near-surface flows in the sun. Astrophys. J. 512, 458 - 470. doi: $10.1086 / 306765$.

Benson, D., Stein, R., Nordlund, Å.: 2006, Supergranulation scale convection simulations. In: Leibacher, J., Stein, R.F., Uitenbroek, H. (eds.) Solar MHD Theory and Observations: A High Spatial Resolution Perspective CS-354, Astron. Soc. Pac., San Francisco, 92.

Birch, A.C., Felder, G.: 2004, Accuracy of the Born and Ray approximations for time-distance helioseismology of flows. Astrophys. J. 616, 1261 - 1264. doi:10.1086/424961.

Birch, A.C., Gizon, L.: 2007, Linear sensitivity of helioseismic travel times to local flows. Astron. Nachr. 328, 228. doi:10.1002/asna.200610724.

Braun, D.C., Birch, A.C., Lindsey, C.: 2004, Local helioseismology of near-surface flows. In: Danesy, D. (ed.) SOHO 14 Helio- and Asteroseismology: Towards a Golden Future SP-559, ESA, Noordwijk, 337 - 340.

Braun, D.C., Birch, A.C., Benson, D., Stein, R.F., Nordlund, Å.: 2007, Helioseismic holography of simulated solar convection and prospects for the detection of small-scale subsurface flows. Astrophys. J. 669, 1395 - 1405. doi: $10.1086 / 521782$.

Couvidat, S., Birch, A.C., Kosovichev, A.G.: 2006, Three-dimensional inversion of sound speed below a sunspot in the Born approximation. Astrophys. J. 640, 516-524. doi:10.1086/500103.

Couvidat, S., Gizon, L., Birch, A.C., Larsen, R.M., Kosovichev, A.G.: 2005, Time-distance helioseismology: inversion of noisy correlated data. Astrophys. J. Suppl. 158, 217-229. doi:10.1086/430423.

Duvall, T.L. Jr., Gizon, L.: 2000, Time - distance helioseismology with $f$ modes as a method for measurement of near-surface flows. Solar Phys. 192, 177-191.

Duvall, T.L. Jr., Jefferies, S.M., Harvey, J.W., Pomerantz, M.A.: 1993, Time-distance helioseismology. Nature 362, 430 - 432. doi:10.1038/362430a0.

Duvall, T.L. Jr., Kosovichev, A.G., Scherrer, P.H., Bogart, R.S., Bush, R.I., de Forest, C., Hoeksema, J.T., Schou, J., Saba, J.L.R., Tarbell, T.D., Title, A.M., Wolfson, C.J., Milford, P.N.: 1997, Time-distance helioseismology with the MDI instrument: initial results. Solar Phys. 170, 63-73.

Gizon, L., Birch, A.C.: 2002, Time-distance helioseismology: the forward problem for random distributed sources. Astrophys. J. 571, 966 - 986. doi:10.1086/340015.

Gizon, L., Birch, A.C.: 2004, Time - distance helioseismology: noise estimation. Astrophys. J. 614, 472 - 489. doi:10.1086/423367.

Gizon, L., Duvall, T.L. Jr., Larsen, R.M.: 2000, Seismic tomography of the near solar surface. J. Astrophys. Astron. 21, 339-342.

Hathaway, D.H., Beck, J.G., Han, S., Raymond, J.: 2002, Radial flows in supergranules. Solar Phys. 205, $25-38$.

Jackiewicz, J., Gizon, L., Birch, A.C.: 2006, Sensitivity of solar $f$-mode travel times to internal flows. In: Lacoste, H. (ed.) SOHO-17. 10 Years of SOHO and Beyond SP-617, ESA, Noordwijk, 38 - 41.

Jackiewicz, J., Gizon, L., Birch, A.C., Duvall, T.L. Jr.: 2007a, Time-distance helioseismology: sensitivity of $f$-mode travel times to flows. Astrophys. J. 671, $1051-1064$. doi:10.1086/522914.

Jackiewicz, J., Gizon, L., Birch, A.C., Thompson, M.J.: 2007b, A procedure for the inversion of $f$-mode travel times for solar flows. Astron. Nachr. 328, 234-239. doi:10.1002/asna.200610725.

Jensen, J.M., Duvall, T.L. Jr., Jacobsen, B.H.: 2003, Noise propagation in inversion of helioseismic timedistance data. In: Sawaya-Lacoste, H. (ed.) GONG+ 2002. Local and Global Helioseismology: the Present and Future SP-517, ESA, Noordwijk, 315-318.

Komm, R., Corbard, T., Durney, B.R., González Hernández, I., Hill, F., Howe, R., Toner, C.: 2004, Solar subsurface fluid dynamics descriptors derived from global oscillation network group and Michelson Doppler imager data. Astrophys. J. 605, 554 - 567. doi:10.1086/382187.

Kosovichev, A.G.: 1996, Tomographic imaging of the Sun's interior. Astrophys. J. 461, L55-L57. doi:10.1086/309989.

Pijpers, F.P., Thompson, M.J.: 1992, Faster formulations of the optimally localized averages method for helioseismic inversions. Astron. Astrophys. 262, L33-L36.

Scherrer, P.H., Bogart, R.S., Bush, R.I., Hoeksema, J.T., Kosovichev, A.G., Schou, J., Rosenberg, W., Springer, L., Tarbell, T.D., Title, A., Wolfson, C.J., Zayer, I.: 1995, MDI Engineering Team: 1995, The solar oscillations investigation - Michelson Doppler imager. Solar Phys. 162, 129-188.

Schmelling, M.: 1995, Averaging correlated data. Phys. Scr. 51, 676-683. 
Woodard, M.F.: 2007, Probing supergranular flow in the solar interior. Astrophys. J. 668, 1189-1195. doi:10.1086/521391.

Zhao, J., Kosovichev, A.G., Duvall, T.L. Jr.: 2001, Investigation of mass flows beneath a sunspot by timedistance helioseismology. Astrophys. J. 557, 384-388. doi:10.1086/321491.

Zhao, J., Georgobiani, D., Kosovichev, A.G., Benson, D., Stein, R.F., Nordlund, Å.: 2007, Validation of time-distance helioseismology by use of realistic simulations of solar convection. Astrophys. J. 659, 848 - 857. doi: $10.1086 / 512009$. 